\title{
A vibrational circular dichroism implementation within a Slater-type-orbital based density functional framework and its application to hexa- and hepta-helicenes
}

\author{
Valentin Paul Nicu - Johannes Neugebauer • \\ Stephen K. Wolff • Evert Jan Baerends
}

Received: 7 October 2006 / Accepted: 14 December 2006 / Published online: 3 February 2007

(C) Springer-Verlag 2007

\begin{abstract}
We describe the implementation of the rotational strengths for vibrational circular dichroism (VCD) in the Slater-type orbital based Amsterdam Density Functional (ADF) package. We show that our implementation, which makes use of analytical derivative techniques and London atomic orbitals, yields origin independent rotational strengths. The basis set dependence in the particular case of Slater-type basis functions is also discussed. It turns out that the triple zeta STO basis sets with one set of polarization functions (TZP) are adequate for VCD calculations. The origindependence of the atomic axial tensors is checked by a distributed origin gauge implementation. The distributed and common origin gauge implementations yield virtually identical atomic axial tensors with the Slatertype basis sets employed here, proving that our implementation yields origin independent rotational
\end{abstract}

Electronic supplementary material The online version of this article (doi:10.1007/s00214-006-0234-x) contains supplementary material, which is available to authorized users.

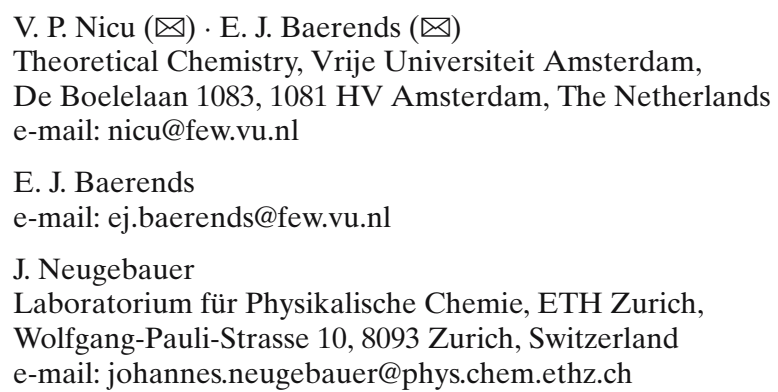

\section{S. K. Wolff}

Nanochemistry Research Institute, Department of Applied Chemistry, Curtin University of Technology, P.O. Box U 1987, Perth, WA 6845, Australia

e-mail: skwolff@iinet.net.au strengths. We verify the implementation for a set of benchmark molecules, for which the dependence of the VCD spectra on the particular choice of the exchangecorrelation functional is studied. The pure functionals BP86 and OLYP show a particularly good performance. Then, we apply this approach to study the VCD spectra of hexa- and hepta- helicenes. In particular we focus on relationships between the sign of the rotational strengths of the two helicenes.

Keywords Vibrational circular dichroism - Atomic axial tensor - VCD spectra

\section{Introduction}

Vibrational circular dichroism (VCD) is the differential absorption of left and right circularly polarized light in a vibrational transition. The VCD intensities are proportional to the rotational strengths which are defined as the imaginary part of the scalar product between the electric and magnetic dipole transition moments.

For exact wave functions the rotational strengths and electric dipole transition moments (EDTM) are originindependent [1]. The magnetic dipole transition moments (MDTM) on the other hand depend on the choice of origin even for exact wave functions. The origin-dependence disappears only after the scalar product of the electric and magnetic dipole transition moments is taken [1] since the origin-dependent part of the MDTM is perpendicular to the EDTM. Within the double harmonic approximation the accuracy of EDTM and MDTM is determined by the accuracy of the harmonic force field, the atomic polar tensor (APT) and the atomic axial tensor (AAT). 
We present here the implementation of Stephens' equations for VCD [2] into the Amsterdam Density Functional program package (ADF, $[3,4]$ ). A combination of analytical derivative techniques is employed for the calculation of harmonic force fields (HFF), APTs and AATs. The HFFs and APTs are calculated using Slater-type orbitals (STO) while London atomic orbitals (LAO) are used for the computation of the AATs.

One of the aims of the paper is to validate the VCD implementation using this specific computational methodology. We demonstrate that the numerical integration used in ADF [5,6] does not affect the origin-independence of the rotational strengths. The common origin gauge is compared with the distributed origin gauge with origins at the equilibrium positions of the nuclei [1]. The accuracy of the calculated AATs, APTs and rotational strengths is assessed via sum rules for a range of systematically increased STO basis sets. We show that even Slater-type basis sets of moderate size yield accurate AATs and rotational strengths. A direct assessment of the obtained VCD spectra in comparison to experiment is carried out for two benchmark molecules in VCD studies, $\alpha$-pinene and Troger's base.

Helicene compounds have been subject to several experimental and theoretical studies using spectroscopic techniques which allow the discrimination between the optical antipodes. They are prototype compounds for the large variety of helical systems occuring in biochemical systems, e.g., DNA, proteins, or amyloid fibrils [7,8]. The investigation of their chiral properties has received much attention [9-11], and only recently an unambiguous assignment of the absolute configuration of heptahelicene could be performed on the basis of VCD spectroscopy [10]. Electronic circular dichroism spectroscopy has been used to prove the enantioselective interaction of particular helicene derivatives with specific forms of DNA [11], which is an important pre-requisite for understanding the mechanisms of anticancer agents. An interesting observation in connection with the chiral properties of screw-shaped molecules is the fact that the chirality of the global helix apparently dominates the chiral properties, even in the presence of different local chirality centers [12]. Therefore, we set out to study the features of the VCD spectra of helicenes for compounds with different numbers of fused aromatic rings. In particular, we focus on the effect of differing normal mode symmetry for normal modes of similar character in hexa- and hepta-helicene.

\section{Theory}

The rotational strength $(R)$ for the fundamental transition $|0\rangle \rightarrow|1\rangle$ of the $i$ th vibrational mode in the elec- tronic ground state is given by [2]:

$R(0 \rightarrow 1)_{i}=\operatorname{Im}\left[\left\langle 0\left|\hat{\mu}_{E}\right| 1\right\rangle_{i} \cdot\left\langle 1\left|\hat{\mu}_{M}\right| 0\right\rangle_{i}\right]$

where $\hat{\mu}_{E}$ and $\hat{\mu}_{M}$ are the electric and magnetic dipole moment operators and $i$ denotes the $i$ th vibrational mode.

Within the harmonic approximation the electric and magnetic dipole transition moments can be written [1]:

$$
\begin{aligned}
\left\langle 0\left|\left(\hat{\mu}_{E}\right)_{\beta}\right| 1\right\rangle_{i} & =\left(\frac{\hbar}{\omega_{i}}\right)^{\frac{1}{2}} \sum_{\lambda \alpha} P_{\alpha \beta}^{\lambda} S_{\lambda \alpha, i} \\
\left\langle 0\left|\left(\hat{\mu}_{M}\right)_{\beta}\right| 1\right\rangle_{i} & =-\left(2 \hbar^{3} \omega_{i}\right)^{\frac{1}{2}} \sum_{\lambda \alpha} M_{\alpha \beta}^{\lambda} S_{\lambda \alpha, i}
\end{aligned}
$$

In Eqs. (2) and (3), $S_{\lambda \alpha, i}$ is the transformation matrix from Cartesian to normal coordinates, $P_{\alpha \beta}^{\lambda}$ and $M_{\alpha \beta}^{\lambda}$ are the atomic polar tensor (APT) and the atomic axial tensor (AAT), respectively, $\hbar$ is the reduced Planck constant, $\omega_{i}$ is the frequency of the $i$ th vibrational mode, $\alpha$ and $\beta$ denote Cartesian coordinates, and $\lambda$ labels the nuclei. Both tensors, APT and AAT, are defined per atom and have electronic and nuclear contributions [1]:

$$
\begin{aligned}
P_{\alpha \beta}^{\lambda} & =E_{\alpha \beta}^{\lambda}+N_{\alpha \beta}^{\lambda} \\
M_{\alpha \beta}^{\lambda} & =I_{\alpha \beta}^{\lambda}+J_{\alpha \beta}^{\lambda}
\end{aligned}
$$

The electronic contributions are

$$
\begin{aligned}
E_{\alpha \beta}^{\lambda} & =\left(\frac{\partial\left\langle\psi_{G}(\mathbf{R})\left|\left(\hat{\mu}_{E}\right)_{\beta}\right| \psi_{G}(\mathbf{R})\right\rangle}{\partial R_{\lambda \alpha}}\right)_{\mathbf{R}^{0}} \\
I_{\alpha \beta}^{\lambda} & =\left\langle\left(\frac{\partial \psi_{G}(\mathbf{R})}{\partial R_{\lambda \alpha}}\right)_{\mathbf{R}^{0}} \mid\left(\frac{\partial \psi_{G}\left(\mathbf{R}^{0}, \mathbf{H}\right)}{\partial H_{\beta}}\right)_{\mathbf{H}=0}\right\rangle
\end{aligned}
$$

while the nuclear ones are:

$$
\begin{aligned}
N_{\alpha \beta}^{\lambda} & =e Z_{\lambda} \delta_{\alpha \beta} \\
J_{\alpha \beta}^{\lambda} & =i \frac{e Z_{\lambda}}{4 \hbar c} \sum_{\gamma} \epsilon_{\alpha \beta \gamma} R_{\lambda \gamma}^{0}
\end{aligned}
$$

In Eqs. (6)-(9), $\psi_{G}$ is the electronic wave function of the ground state $G, Z_{\lambda}$ and $\mathbf{R}_{\lambda}$ are the charge and the position of nucleus $\lambda, \mathbf{R}_{\lambda}^{0}$ is $\mathbf{R}_{\lambda}$ at the equilibrium geometry, $\mathbf{H}$ is a static magnetic field, $\epsilon_{\alpha \beta \gamma}$ is the Levi-Civita tensor with the indices $\alpha, \beta, \gamma$ running over Cartesian components, $e$ is the elementary charge and $c$ is the speed of light in vacuum. Note that in Eq. (7), $\psi_{G}(\mathbf{R})$ depends on the positions of the nuclei, which are not fixed, while $\psi_{G}\left(\mathbf{R}^{0}, \mathbf{H}\right)$ has to be calculated for the equilibrium positions of the nuclei in the presence of a magnetic field. To generalize, with regard to the notation used in this paper, a quantity $\Gamma$ depends on a parameter/perturbation $y$ only when it is written as $\Gamma(y)$. Otherwise it refers to the unperturbed case. 
The APTs are origin-independent [1]. The AATs, on the other hand, are origin-dependent due to their dependence on the magnetic field. The origin-dependence of the AATs is given by [1]:

$\left(M_{\alpha \beta}^{\lambda}\right)^{\mathbf{o}_{1}}=\left(M_{\alpha \beta}^{\lambda}\right)^{\mathbf{o}_{2}}+\frac{i}{4 \hbar c} \sum_{\gamma \delta} \epsilon_{\beta \gamma \delta} Y_{\gamma} P_{\alpha \delta}^{\lambda}$

where $\mathbf{O}_{1}$ and $\mathbf{O}_{2}$ are the position vectors of the two different choices of the origin of the coordinate system while $\mathbf{Y}$ is the displacement vector between the two origins $\left(\mathbf{Y}=\mathbf{O}_{2}-\mathbf{O}_{1}\right)$. Note that the calculated rotational strengths are origin independent only if the calculated AATs and APTs fulfill Eq. (10) exactly [1].

By defining the following tensors:

$\Sigma_{\alpha \beta}^{1}=4 \hbar c \sum_{\lambda} \operatorname{Im}\left(M_{\alpha \beta}^{\lambda}\right)$

$\Sigma_{\alpha \beta}^{2}=\sum_{\lambda \gamma \delta} \epsilon_{\beta \gamma \delta} R_{\lambda \gamma}^{0} P_{\alpha \delta}^{\lambda}$

$\Sigma_{\alpha \beta}^{3}=\sum_{\gamma} \epsilon_{\alpha \beta \gamma}\left(\mu^{G}\right)_{\gamma}$

$\Sigma_{\alpha \beta}^{4}=\sum_{\lambda} P_{\alpha \beta}^{\lambda}$

it can be shown that the APTs and AATs are further interconnected via sum rules. In Eq. (13), $\mu^{G}$ is the dipole moment of the ground state. For neutral systems and exact wave functions these tensors obey the following sum rules [13]:

$\Sigma_{\alpha \beta}^{1}=\Sigma_{\alpha \beta}^{2}=\Sigma_{\alpha \beta}^{3}, \quad \Sigma_{\alpha \beta}^{4}=0$

\section{Implementation}

\subsection{Methodology}

We start by writing the APTs and AATs in terms of Kohn-Sham molecular orbitals (MO). After introducing the coupled-perturbed Kohn-Sham equations (CPKS) the final expressions for APTs and AATs are derived and their implementation is discussed. Only the electronic contributions will be considered since the implementation of the nuclear parts, Eqs. (8) and (9), is trivial.

In the case when the electronic wave function, $\psi_{G}$, is approximated by a single Slater determinant, Eqs. (6) and (7) become [14]:

$$
\begin{aligned}
E_{\alpha \beta}^{\lambda} & =\left(\frac{\partial}{\partial R_{\lambda \alpha}} \int \mathbf{r}_{\beta} \rho \mathrm{d} \mathbf{r}\right)_{\mathbf{R}^{\mathbf{0}}} \\
& =\int \mathbf{r}_{\beta}\left(\frac{\partial \rho}{\partial R_{\lambda \alpha}}\right)_{\mathbf{R}^{\mathbf{0}}} \mathrm{d} \mathbf{r}=\int \mathbf{r}_{\beta} \rho^{(1)} \mathrm{d} \mathbf{r} \\
I_{\alpha \beta}^{\lambda} & =2 \sum_{j=1}^{\mathrm{N}_{\text {occ }}}\left\langle\left(\frac{\partial \varphi_{j}}{\partial R_{\lambda \alpha}}\right)_{\mathbf{R}^{\mathbf{0}}} \mid\left(\frac{\partial \varphi_{j}}{\partial H_{\beta}}\right)_{\mathbf{H}=\mathbf{0}}\right\rangle
\end{aligned}
$$

with:

$\rho=2 \sum_{j=1}^{N_{\text {occ }}} \varphi_{j} \varphi_{j}^{*}$ and $\rho^{(1)}=2 \sum_{j=1}^{N_{\text {occ }}}\left(\varphi_{j}^{(1)} \varphi_{j}^{*}+\varphi_{j} \varphi_{j}^{(1)^{*}}\right)$

Here, $\varphi_{j}$ is the $j$ th MO, $\rho$ and $\rho^{(1)}$ are the electron density and its derivative with respect to a nuclear displacement, respectively, $\mathbf{r}$ is a spatial coordinate, $j$ runs over the occupied MOs, and $N_{\text {occ }}$ is the number of occupied MOs. Note that Eqs. (17) and (18) are written for the case of a closed-shell system. Throughout this work the upper index "(1)" denotes first order derivatives with respect to some perturbation parameter $y$.

According to the CPKS formalism the first order MO derivatives with respect to a perturbation $y$ can be written as [15,16]:

$\varphi^{(1)}=\chi^{(1)} \mathbf{C}+\varphi \mathbf{U}^{(1)}$

In Eq. (19) $\varphi$ is a row vector containing the MOs, $\chi$ is a row vector containing the perturbation-dependent basis functions and $\mathbf{U}$ is the unitary transformation that relates the perturbed eigenvectors to the unperturbed ones $[15,16]$ :

$\mathbf{C}(y)=\mathbf{C U}(y)$

The $\mathbf{U}^{(1)}$ matrix required in Eq. (19) is calculated from the CPKS equations $[15,16]$ :

$U_{i j}^{(1)}=\frac{F_{i j}^{(1)}-\epsilon_{j} S_{i j}^{(1)}}{\epsilon_{j}-\epsilon_{i}}(i \neq j), \quad U_{i i}^{(1)}=-\frac{1}{2} S_{i i}^{(1)}$

$U_{i j}^{(1)}+U_{j i}^{(1)^{*}}+S_{i j}^{(1)}=0$

where $\mathbf{S}$ and $\mathbf{F}$ are the overlap and Fock matrices in the MO basis, respectively, and $\epsilon_{i}, \epsilon_{j}$ denote orbital energies.

\subsection{The atomic axial tensor}

The electronic contribution to the atomic axial tensor, Eq. (17), is an overlap integral of two first-order perturbed wave functions. One perturbation is due to a nuclear displacement, the other is due to the magnetic field. 
From Eq. (19) the derivatives of the $j$ th MO can be written:

$\varphi_{j}^{(1)}=\sum_{a=1}^{N_{\mathrm{bas}}} \chi_{a}^{(1)} C_{a j}+\sum_{i=1}^{N_{\mathrm{MO}}} \varphi_{i} U_{i j}^{(1)}$

where $N_{\text {bas }}$ is the number of basis functions and $N_{\mathrm{MO}}$ is the number of MOs.

By inserting Eq. (23) for magnetic and nuclear displacement perturbations into Eq. (17) we obtain:

$$
\begin{aligned}
I_{\alpha \beta}^{\lambda}= & \sum_{j=1}^{N_{\text {occ }}} \sum_{a, b=1}^{N_{\text {bas }}} C_{a j} C_{b j}\left\langle\chi_{a}^{(1), R_{\lambda \alpha}} \mid \chi_{b}^{(1), H_{\beta}}\right\rangle \\
& +\sum_{j=1}^{N_{\text {occ }}} \sum_{i^{\prime}=1}^{N_{\text {MO }}} \sum_{a=1}^{N_{\text {bas }}} C_{a j} U_{i^{\prime} j}^{(1), H_{\beta}}\left\langle\chi_{a}^{(1), R_{\lambda \alpha}} \mid \varphi_{i^{\prime}}\right\rangle \\
& +\sum_{j=1}^{N_{\text {occ }}} \sum_{i=1}^{N_{\text {MO }}} \sum_{b=1}^{N_{\text {bas }}} U_{i j}^{(1), R_{\lambda \alpha}} C_{b j}\left\langle\varphi_{i} \mid \chi_{b}^{(1), H_{\beta}}\right\rangle \\
& +\sum_{j=1}^{N_{\text {occ }}} \sum_{i, i^{\prime}=1}^{N_{\text {MO }}} U_{i j}^{(1), R_{\lambda \alpha}} U_{i^{\prime} j}^{(1), H_{\beta}}\left\langle\varphi_{i} \mid \varphi_{i^{\prime}}\right\rangle
\end{aligned}
$$

In Eq. (24) the upper indexes $R_{\lambda \alpha}$ and $H_{\beta}$ label the nuclear displacement and magnetic field perturbations with respect to which the derivatives were taken. By writing the MOs as linear combinations of basis functions and using their orthogonality, the final expression for $I_{\alpha \beta}^{\lambda}$ is obtained:

$$
\begin{aligned}
I_{\alpha \beta}^{\lambda}= & \sum_{j=1}^{N_{\text {occ }}} \sum_{a, b=1}^{N_{\text {bas }}} C_{a j} C_{b j}\left\langle\chi_{a}^{(1), R_{\lambda \alpha}} \mid \chi_{b}^{(1), H_{\beta}}\right\rangle \\
& +\sum_{j=1}^{N_{\text {occ }}} \sum_{i=1}^{N_{\mathrm{MO}}} \sum_{a, b=1}^{N_{\mathrm{bas}}} C_{a j} C_{b i} U_{i j}^{(1), H_{\beta}}\left\langle\chi_{a}^{(1), R_{\lambda \alpha}} \mid \chi_{b}\right\rangle \\
& +\sum_{j=1}^{N_{\text {occ }}} \sum_{i=1}^{N_{\mathrm{MO}}} \sum_{a, b=1}^{N_{\text {bas }}} C_{a i} C_{b j} U_{i j}^{(1), R_{\lambda \alpha}}\left\langle\chi_{a} \mid \chi_{b}^{(1), H_{\beta}}\right\rangle \\
& +\sum_{j=1}^{N_{\text {occ }}} \sum_{i=1}^{N_{\mathrm{MO}}} U_{i j}^{(1), R_{\lambda \alpha}} U_{i j}^{(1), H_{\beta}}
\end{aligned}
$$

As can be seen from Eq. (25) the calculation of the electronic contribution of the AATs requires knowledge of the unitary matrices $\mathbf{U}^{(1), R_{\lambda \alpha}}$ and $\mathbf{U}^{(1), H_{\beta}}$, including their occupied-occupied (occ-occ) blocks and the unperturbed eigenvectors, C. The integrals $\left\langle\chi_{a}^{(1), R_{\lambda \alpha}}\right|$ $\left.\chi_{b}^{(1), H_{\beta}}\right\rangle,\left\langle\chi_{a}^{(1), R_{\lambda \alpha}} \mid \chi_{b}\right\rangle$, and $\left\langle\chi_{a} \mid \chi_{b}^{(1), H_{\beta}}\right\rangle$ are calculated numerically using London atomic orbitals (LAOs) as basis functions. An LAO is obtained from a field independent basis function by adding a phase factor that depends on the magnetic field [17-20]:

$\sigma_{a}^{A}(\mathbf{r})=\chi_{a}^{A}(\mathbf{r}) \cdot \exp \left[-i \frac{e}{\hbar c}\left(\mathbf{H} \times \mathbf{R}_{A}\right) \mathbf{r}\right]$

where $A$ labels the nucleus on which the basis function $\chi_{a}^{A}$ is centered, $\mathbf{H}$ is a static magnetic field and $\mathbf{R}_{A}$ is the position vector of the $A$ th nucleus. Note that in our case $\chi_{a}^{A}$ is an STO.

For a zero magnetic field the LAOs reduce to STOs and therefore only quantities directly related to a magnetic field $\left(U_{i^{\prime} j}^{(1), H_{\beta}}\right.$ and $\left.\chi_{b}^{(1), H_{\beta}}\right)$ are calculated using LAOs. The calculation of $\mathbf{U}^{(1), H_{\beta}}$ and $\mathbf{U}^{(1), R_{\lambda \alpha}}$ requires the solution of the CPKS equations for magnetic-field perturbations and nuclear displacements, respectively. In general, the CPKS equations, Eqs. (21) and (22), have to be solved in an iterative manner due to the fact that the $\mathbf{U}^{(1)}$ matrix depends on the first order perturbed Fock matrix, $\mathbf{F}^{(1)}$, which depends on the first order density, $\rho^{(1)}$, which in turn depends on the $\mathbf{U}^{(1)}$ matrix. For magnetic perturbations however, the first order density is zero (see Appendix 1 for more details), thus $\mathbf{U}^{(1), H_{\beta}}$ can be calculated directly from Eq. (21).

As mentioned, the occ-occ blocks of both the nuclear displacement and magnetic perturbation $\mathbf{U}^{(1)}$ matrices are present in the expression for $I_{\alpha \beta}^{\lambda}$. This is because the basis sets depend on the perturbation. For degenerate orbitals, the denominator in the expression for off-diagonal occ-occ matrix elements in Eq. (21) will diverge. However, as is shown in Appendix 2 the contributions from the off-diagonal matrix elements of the occ-occ blocks can be expressed in terms of the perturbed overlap matrices and thus avoid this complication.

\subsection{The atomic polar tensor}

In order to calculate the APT components of Eq. (4) we need to calculate the electronic contributions $E_{\alpha \beta}^{\lambda}$ of Eq. (16). These in turn depend on $\rho^{(1)}$ due to a nuclear displacement perturbation. Inserting Eq. (23) into Eq. (18), and making use of the condition Eq. (22) we obtain:

$$
\begin{aligned}
\rho^{(1), R_{\lambda \alpha}}= & \sum_{i=1}^{N_{\text {vir }}} \sum_{j=1}^{N_{\text {occ }}}\left(U_{i j}^{(1), R_{\lambda \alpha}{ }^{*}} \varphi_{i}^{*} \varphi_{j}+U_{i j}^{(1), R_{\lambda \alpha}} \varphi_{i} \varphi_{j}^{*}\right) \\
& +\sum_{j=1}^{N_{\text {occ }}} \sum_{a=1}^{N_{\text {bas }}}\left(C_{a j} \varphi_{j}^{*}+C_{a j}^{*} \varphi_{j}\right) \chi_{a}^{(1), R_{\lambda \alpha}} \\
& -\sum_{i=1}^{N_{\text {occ }}} \sum_{j=1}^{N_{\text {occ }}} S_{j i}^{(1), R_{\lambda \alpha}} \varphi_{i}^{*} \varphi_{j}
\end{aligned}
$$


Inserting this into Eq. (16) gives

$$
\begin{aligned}
E_{\alpha \beta}^{\lambda}= & \sum_{i=1}^{N_{\text {vir }}} \sum_{j=1}^{N_{\text {occ }}} U_{i j}^{(1), R_{\lambda \alpha}{ }^{*}}\left\langle\varphi_{i}\left|\mathbf{r}_{\beta}\right| \varphi_{j}\right\rangle \\
& +\sum_{i=1}^{N_{\text {vir }}} \sum_{j=1}^{N_{\text {occ }}} U_{i j}^{(1), R_{\lambda \alpha}}\left\langle\varphi_{j}\left|\mathbf{r}_{\beta}\right| \varphi_{i}\right\rangle \\
& +\sum_{j=1}^{N_{\text {occ }}} \sum_{a=1}^{N_{\text {bas }}} C_{a j}^{*}\left\langle\chi_{a}^{(1), R_{\lambda \alpha}}\left|\mathbf{r}_{\beta}\right| \varphi_{j}\right\rangle \\
& +\sum_{j=1}^{N_{\text {occ }}} \sum_{a=1}^{N_{\text {bas }}} C_{a j}\left\langle\varphi_{j}\left|\mathbf{r}_{\beta}\right| \chi_{a}^{\left.(1), R_{\lambda \alpha}\right\rangle}\right. \\
& -\sum_{i=1}^{N_{\text {occ }}} \sum_{j=1}^{N_{\text {occ }}} S_{j i}^{(1), R_{\lambda \alpha}}\left\langle\varphi_{i}\left|\mathbf{r}_{\beta}\right| \varphi_{j}\right\rangle
\end{aligned}
$$

\subsubsection{Calculating $\mathbf{U}^{(\mathbf{1}), \mathbf{R}_{\lambda \alpha}}$ for the APT}

The one-electron Kohn-Sham operator is:

$$
\begin{aligned}
h & =T+V_{N e}+V_{C}+V_{X C} \\
& =-\frac{p^{2}}{2}-\sum_{A} \frac{Z_{A}}{\left|\mathbf{r}-\mathbf{R}_{A}\right|}+\int \frac{\rho}{\left|\mathbf{r}-\mathbf{r}^{\prime}\right|} \mathrm{d} \mathbf{r}^{\prime}+V_{X C}
\end{aligned}
$$

where the first term is the kinetic energy, the second is the nuclear potential, the third is the electronic Coulomb potential, and the final term is the exchange-correlation potential. The derivative of this with respect to a nuclear displacement, $R_{\lambda \alpha}$, is:

$h^{(1), R_{\lambda \alpha}}=\frac{\partial V_{N e}}{\partial R_{\lambda \alpha}}+\int \frac{\rho^{(1), R_{\lambda \alpha}}}{\left|\mathbf{r}-\mathbf{r}^{\prime}\right|} \mathrm{d} \mathbf{r}^{\prime}+\frac{\partial V_{X C}}{\partial R_{\lambda \alpha}}$

Inserting Eq. (27) into the above expression gives an expression for $h^{(1), R_{\lambda \alpha}}$ that can be split into a part that contains the $U^{(1), R_{\lambda, \alpha}}$ and a part that does not, so that $F_{i j}^{(1), R_{\lambda \alpha}}$ of Eq. (21) can be written as:

$F_{i j}^{(1), R_{\lambda \alpha}}=F_{i j}^{\left(U^{1}\right)}+F_{i j}^{\left(\mathrm{non}-U^{1}\right)}$
Putting this into Eq. (21) gives

$U_{i j}^{(1), R_{\lambda \alpha}}=\frac{F_{i j}^{\left(U^{1}\right)}}{\epsilon_{j}-\epsilon_{i}}+\frac{F_{i j}^{\left(\mathrm{non}-U^{1}\right)}-\epsilon_{i} S_{i j}^{(1), R_{\lambda \alpha}}}{\epsilon_{j}-\epsilon_{i}}$

This equation has the "matrix" form

$\mathbf{u}=\mathbf{A}(\mathbf{u})+\mathbf{v}$,

where $\mathbf{u}$ is a vector with components $U_{i j}^{(1)}$, the matrix $\mathbf{A}$ depends on $\mathbf{u}$, and $\mathbf{v}$ is a vector free of the $U_{i j}^{(1)}$. It is solved using an iterative method [15,16,21].

\section{Validation for a set of benchmark molecules}

The theory presented in Sects. 2 and 3 has been implemented in the ADF program. The AATs, $\mathbf{U}^{(1), H_{\beta}}$ and rotational strengths are calculated with the newly developed VCD module of ADF, while the APTs, $\mathbf{U}^{(1), R_{\lambda}}$ and $\mathbf{S}_{\lambda \alpha, i}$ are calculated with the existing second derivatives (SD) module [21]. The validation of the implementation is done in two ways. First, using Eqs. (10) and (15) we examine the accuracy of the calculated tensors using the ethylene oxide (oxirane) (1) as our test molecule. Second, we compare calculated rotational strengths with the experimental values for two benchmark molecules: $\alpha$-pinene (2) and Troger's base (3) (Fig. 1).

The calculations were performed using three different exchange-correlation functionals BP86 [22,23], OLYP $[24,25]$, and BLYP $[22,26]$ and basis sets from the ADF basis set library. The basis sets used were SZ, DZ, DZP, TZP, TZ2P and ET-pVQZ [27]. The geometries were optimized with ADF for each choice of basis set and functional.

\subsection{The atomic axial tensors}

\subsubsection{Gauge dependence}

Rotational strengths should be origin-independent. Historically this has been an issue [20,28-31]. Initially, the importance of using London atomic orbitals was not

Fig. 1 Test molecules used to verify the VCD implementation. Oxirane (1); $\alpha$-pinene (2); Troger's base (3)

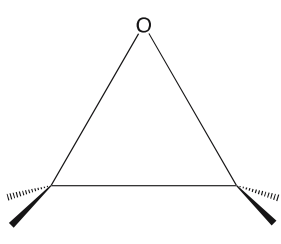

(1)

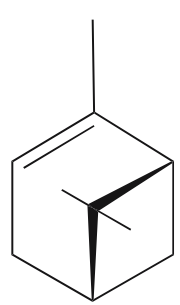

(2)

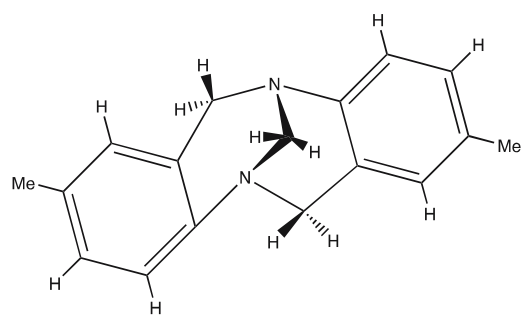

(3) 
fully recognized. The failure to use LAOs combined with the use of basis sets of limited size and/or different levels of theory for the computation of the AATs, APTs and force fields yielded rotational strengths that were origin-dependent. As an attempt to remedy this problem the distributed origin (DO) gauge was introduced [1]. In the DO gauge the AAT of each nucleus is calculated with respect to an origin centred at that nucleus. Then using the shift formula of Eq. (10), they are referred to a common origin $\mathbf{O}_{1}$. Since this does not yield correct results when LAO's are not used, the results still prove to be dependent on the choice of origin $\mathbf{O}_{1}$, although they generally yielded results of superior accuracy compared to the ones obtained when using the common origin $(\mathrm{CO})$ gauge $[28,29]$.

Bak et al. [20] have proven that if LAOs are used, the CO and DO gauges yield identical AATs at any basis set level and therefore origin-independent rotational strengths. Still, numerical error sources, such as numerical integration of STO-based integrals and/or the limited accuracy of $\mathbf{U}^{(1)}$ matrices may prevent perfect independency of the choice of origin. We therefore verify the degree of origin-independency of our implementation by calculating the AATs with respect to the $\mathrm{CO}$ gauge and the DO gauge with origins at nuclei. In the $\mathrm{CO}$ gauge the AATs are calculated from Eq. (5) using Eqs. (7) and (9); the origin of the coordinate system is at the molecular center of mass $(\mathrm{CM})$. In the DO gauge with origins at the nuclei, the AATs are calculated with respect to the $\mathrm{CM}$ from the following equation:

$$
\left(M_{\alpha \beta}^{\lambda}\right)^{\mathbf{Y}_{C M}}=\left(M_{\alpha \beta}^{\lambda}\right)^{\mathbf{R}_{\lambda}^{0}}+\frac{i}{4 \hbar c} \sum_{\gamma \delta} \epsilon_{\beta \gamma \delta}\left(\mathbf{R}_{\lambda}^{0}-\mathbf{Y}_{C M}\right)_{\gamma} P_{\alpha \delta}^{\lambda}
$$

Equation (34) is obtained from Eq. (10) by choosing $\mathbf{O}_{2}=\mathbf{R}_{\lambda}^{0}$ and $\mathbf{O}_{1}=\mathbf{Y}_{C M}$, where $\mathbf{Y}_{C M}$ is the position vector of the center of mass.

Since $\mathbf{U}^{(1), H_{\beta}}$, required for the calculation of AATs, is origin-dependent, the solution of the CPKS equations for a magnetic field perturbation has to be repeated for each intermediate origin $\mathbf{R}_{\lambda}^{0}$ when DO is used. The SCF and the CPKS calculations for nuclear displacement are origin-independent and therefore do not have to be repeated. Even though solving the CPKS equations for a magnetic field perturbation is much simpler than solving them for a nuclear displacement perturbation (which is the most time consuming part of the calculation of AATs), the calculation of $\mathbf{U}^{(1), H_{\beta}}$ in the DO gauge can become the bottleneck for large molecules. Therefore, it is desirable to use only the $\mathrm{CO}$ gauge for the calculations of the AATs.
Table 1 Comparison of AATs of oxirane (1) calculated using CO and DO gauges and the BP86 functional

\begin{tabular}{|c|c|c|c|c|c|c|}
\hline \multirow{2}{*}{$\begin{array}{l}\text { Basis set } \\
M_{\alpha \beta}^{\lambda}\end{array}$} & \multicolumn{6}{|c|}{ SZ } \\
\hline & \multicolumn{3}{|c|}{$\left(M_{\alpha \beta}^{\lambda}\right)^{C M, C O}$} & \multicolumn{3}{|c|}{$\left(M_{\alpha \beta}^{\lambda}\right)^{C M, D O}$} \\
\hline \multirow{4}{*}{$M_{\alpha \beta}^{1}$} & 0.0000 & -0.2190 & 0.0001 & 0.0000 & -0.2190 & 0.0001 \\
\hline & -0.0759 & 0.0000 & 0.0000 & -0.0759 & 0.0000 & 0.0000 \\
\hline & -0.0001 & 0.0001 & 0.0002 & -0.0001 & 0.0001 & 0.0002 \\
\hline & 0.0002 & 0.0656 & -0.0002 & 0.0002 & 0.0656 & -0.0002 \\
\hline \multirow[t]{3}{*}{$M_{\alpha \beta}^{2}$} & -0.0390 & 0.0000 & 0.0383 & -0.0389 & 0.0000 & 0.0383 \\
\hline & 0.0004 & 0.0555 & -0.0004 & 0.0004 & 0.0555 & -0.0004 \\
\hline & -0.0438 & 0.0080 & 0.0401 & -0.0439 & 0.0080 & 0.0401 \\
\hline \multirow[t]{2}{*}{$M_{\alpha \beta}^{4}$} & 0.0381 & 0.0046 & 0.0421 & 0.0381 & 0.0046 & 0.0421 \\
\hline & -0.0757 & -0.0164 & 0.0686 & -0.0757 & -0.0164 & 0.0686 \\
\hline \multicolumn{3}{|l|}{ Basis set } & \multicolumn{3}{|c|}{ ET-pVQZ } & \\
\hline$M_{\alpha \beta}^{\lambda}$ & \multicolumn{3}{|c|}{$\left(M_{\alpha \beta}^{\lambda}\right)^{C M, C O}$} & \multicolumn{3}{|c|}{$\left(M_{\alpha \beta}^{\lambda}\right)^{C M, D O}$} \\
\hline \multirow{4}{*}{$M_{\alpha \beta}^{1}$} & 0.0000 & -0.7831 & 0.0000 & 0.0000 & -0.7830 & 0.0000 \\
\hline & 0.1832 & -0.0000 & -0.0000 & 0.1831 & -0.0000 & -0.0000 \\
\hline & -0.0000 & -0.0000 & 0.0000 & -0.0000 & -0.0000 & 0.0000 \\
\hline & -0.0000 & 0.0891 & -0.0000 & -0.0000 & 0.0890 & -0.0000 \\
\hline \multirow[t]{2}{*}{$M_{\alpha \beta}^{2}$} & -0.0766 & 0.0000 & 0.2604 & -0.0766 & 0.0000 & 0.2604 \\
\hline & 0.0000 & -0.0033 & -0.0000 & 0.0000 & -0.0034 & -0.0000 \\
\hline \multirow{3}{*}{$M_{\alpha \beta}^{4}$} & -0.0114 & 0.1052 & 0.0118 & -0.0114 & 0.1052 & 0.0118 \\
\hline & 0.0354 & 0.0150 & 0.0534 & 0.0354 & 0.0150 & 0.0534 \\
\hline & -0.1074 & 0.0485 & 0.0024 & -0.1075 & 0.0486 & 0.0024 \\
\hline
\end{tabular}

$M_{\lambda \beta}^{\alpha}$ are given in a.u

Table 1 presents the AATs calculated using the CO, $\left(M_{\alpha \beta}^{\lambda}\right)^{C M, C O}$, and DO, $\left(M_{\alpha \beta}^{\lambda}\right)^{C M, D O}$, gauges with SZ and ET-pVQZ basis sets for the oxirane molecule. For brevity, only the results for the smallest (SZ) and largest (ET-pVQZ) basis sets and the BP86 functional are shown.

As can be seen the CO and DO gauges yield virtually identical AATs. Thus, Eq. (10) is fulfilled for all basis sets which proves that our implementation yields originindependent rotational strengths [1]. Therefore, the CO gauge is the method of choice for VCD calculation using analytical derivatives and LAOs. The DO gauge on the other hand can be used as an additional check for the correctness and accuracy of the code.

\subsubsection{Sum rules}

A systematic study of the accuracy of the calculated AATs is difficult due to their large number ( $9 \mathrm{~N}$ tensor components for a molecule with $\mathrm{N}$ atoms). A convenient measure of the quality of the AATs is the extent to which they satisfy the sum rules of Eqs. (15). The convergence to a stable limit and the degree to which the sum rules are fulfilled is studied by systematically increasing the size of the basis sets. 
Table 2 Comparison of $\bar{\Sigma}_{x y}^{1}=\frac{1}{2}\left(\Sigma_{x y}^{1}-\Sigma_{y x}^{1}\right)$ and $\Sigma_{x y}^{2}$ for $\mathbf{1}$

\begin{tabular}{|c|c|c|c|c|c|c|c|c|c|c|c|c|}
\hline \multirow{2}{*}{$\begin{array}{l}\text { Functional } \\
\text { Basis set }\end{array}$} & \multicolumn{4}{|c|}{ BP86 } & \multicolumn{4}{|c|}{ OLYP } & \multicolumn{4}{|c|}{ BLYP } \\
\hline & $a$ & $\bar{\Sigma}_{x y}^{1}$ & $\Sigma_{x y}^{2}$ & $b$ & $a$ & $\bar{\Sigma}_{x y}^{1}$ & $\Sigma_{x y}^{2}$ & $b$ & $a$ & $\bar{\Sigma}_{x y}^{1}$ & $\Sigma_{x y}^{2}$ & $b$ \\
\hline $\mathrm{SZ}$ & 83.8 & 0.115 & 0.476 & 75.8 & 81.0 & 0.129 & 0.473 & 72.7 & 85.3 & 0.110 & 0.431 & 74.4 \\
\hline DZ & 38.0 & 0.983 & 1.035 & 5.0 & 45.8 & 0.995 & 1.033 & 3.6 & 28.3 & 0.961 & 1.036 & 7.2 \\
\hline DZP & 5.1 & 0.749 & 0.716 & 4.6 & 10.2 & 0.752 & 0.698 & 7.7 & 1.3 & 0.759 & 0.727 & 4.4 \\
\hline TZP & 4.6 & 0.745 & 0.740 & 0.6 & 4.8 & 0.715 & 0.696 & 2.7 & 2.0 & 0.764 & 0.766 & 0.2 \\
\hline TZ2P & 4.2 & 0.742 & 0.732 & 1.3 & 3.8 & 0.708 & 0.686 & 3.2 & 1.3 & 0.759 & 0.758 & 0.1 \\
\hline ET-pVQZ & - & 0.712 & 0.717 & 0.6 & - & 0.682 & 0.679 & 0.4 & - & 0.749 & 0.749 & 0.0 \\
\hline Exp. $\Sigma_{x y}^{3}$ & \multicolumn{4}{|c|}{$0.7396 \pm 0.0039$} & \multicolumn{4}{|c|}{$0.7396 \pm 0.0039$} & \multicolumn{4}{|c|}{$0.7396 \pm 0.0039$} \\
\hline
\end{tabular}

$a$ denotes the deviation from the ET-pVQZ value; $b$ denotes the deviations of $\bar{\Sigma}_{x y}^{1}$ from $\Sigma_{x y}^{2} ; \bar{\Sigma}_{x y}^{1}, \Sigma_{x y}^{2}$ and $\Sigma_{x y}^{3}$ are given in a.u.; $a$ and $b$ are given in percent. The experimental value for $\Sigma_{x y}^{3}=\mu_{z}^{G}[32]$ is also shown. In the basis set limit $\bar{\Sigma}_{x y}^{1}=\Sigma_{x y}^{2}=\Sigma_{x y}^{3}$

With the $C_{2}$-axis chosen as the $Z$-axis, the only nonzero tensor components of oxirane are $\Sigma_{x y}^{i}=-\Sigma_{y x}^{i}$ $(i=1,2,3)$. The sum rules for the APTs, $\Sigma_{x y}^{2}=\Sigma_{x y}^{3}$ and $\Sigma_{x y}^{4}=0$ (not shown) are satisfied for all basis sets. In this context, $\Sigma_{x y}^{1}=\Sigma_{x y}^{2}$, relating the AATs and APTs, is the only sum rule of interest. In Table 2 , the arithmetic mean of $\Sigma_{x y}^{1}$ and $-\Sigma_{y x}^{1}$ denoted as $\bar{\Sigma}_{x y}^{1}, \Sigma_{x y}^{2}$ and the experimental value of $\Sigma_{x y}^{3}$ are shown.

As can be seen from Table 2, the magnitude of $\bar{\Sigma}_{x y}^{1}$ and $\Sigma_{x y}^{2}$ converge to a stable limit as the basis set size is increased. The average deviations of TZP and TZ2P values from the ET-pVQZ are 3.82 and $3.11 \%$, respectively, for all three functionals. The differences between $\bar{\Sigma}_{x y}^{1}$ and $\Sigma_{x y}^{2}$ are less than $1 \%$ for ET-pVQZ and around $2 \%$ for TZP and TZ2P for all three functionals. Therefore, it is reasonable to assume that the ET-VQZ results are good approximations of the basis set limit. As far as the differences between different functionals are concerned we note that for oxirane the AATs obtained with BLYP converge faster and fulfill the sum rules to a higher degree then the ones calculated with BP86 and OLYP.

The AATs are not directly measurable. However, they are connected via sum rules to the electric dipole moment which is experimentally accessible. Since the AATs are very sensitive to the functional used we compare the ET-pVQZ value of $\bar{\Sigma}_{x y}^{1}$ obtained with all three functionals to the experimental value of $\Sigma_{x y}^{3}$. As can be seen in Table 2 the BLYP, BP86 and OLYP values deviate from the experimental value by 1.2, 3.7 and $8.9 \%$, respectively. Again, the BLYP functional yields AATs of better accuracy than the BP86 and OLYP. Thus, for oxirane, the relative accuracy of the functionals considered here for the calculation of the AATs is BLYP > BP86 > OLYP. However, the accuracy of the vibrational rotational strength is a function not only of the accuracy of the AATs, but also of the accuracy of the APTs and the normal modes, characterized by the transformation $\mathbf{S}_{\lambda \alpha, i}$. In fact, Stephens et al. [32] has shown that the accuracy of the harmonic force field (which determines $\mathbf{S}_{\lambda \alpha, i}$ ) is the most critical parameter. Therefore, when comparing calculated VCD intensities to experiment the relative accuracy of different functionals may be different from the relative accuracy of the AATs obtained with the considered functionals.

\subsection{The rotational strengths}

To study the quality of the VCD spectra obtained with our implementation we calculated the rotational strengths for $\alpha$-pinene (2) and Troger's base (3). For $\mathbf{2}$ the calculations were performed using the BP86, BLYP and OLYP functionals and TZP and ET-pVQZ basis sets. In the case of $\mathbf{3}$, BP86 and OLYP in combination with the TZP basis were used. The VCD spectra are simulated by representing the peaks as Lorentzian bands [33] with a constant half width of $4 \mathrm{~cm}^{-1}$. In all VCD spectra, the differential intensities $(\Delta \epsilon)$ are in units of molar absorptivity $\left(\mathrm{L} \mathrm{mol}^{-1} \mathrm{~cm}^{-1}\right)$.

\subsubsection{VCD spectra of $\alpha$-pinene (2)}

The calculated and experimental spectra of $\mathbf{2}$ are shown in Figs. 2 and 3. The experimental data are taken from Ref. [33]. The calculated and experimental frequencies and rotational strengths are shown in Tables 1 and 2 from the electronic supplementary material (see ESM).

As can be seen in Figs. 2 and 3 the OLYP and BP86 functionals yield frequencies that are in very good agreement with the experiment even without scaling. It is a well-known fact that harmonic frequencies from BP86 are usually close to experimental fundamental frequencies due to an error cancellation effect [34-36]. The frequencies calculated using BLYP are in fair agreement with the experiment although not as good as the ones obtained with OLYP and BP86. Since the three functionals give frequencies that are fairly close, and also yield rotational strengths that are qualitatively similar, 
Fig. 2 VCD spectra for $\alpha$-pinene (2). Comparison of experiment with ET-pVQZ basis calculations for different functionals (BP86, OLYP, BLYP)

Fig. 3 VCD spectra for $\alpha$-pinene (2). Comparison of experiment with TZP basis calculations for different functionals (BP86, OLYP, BLYP)
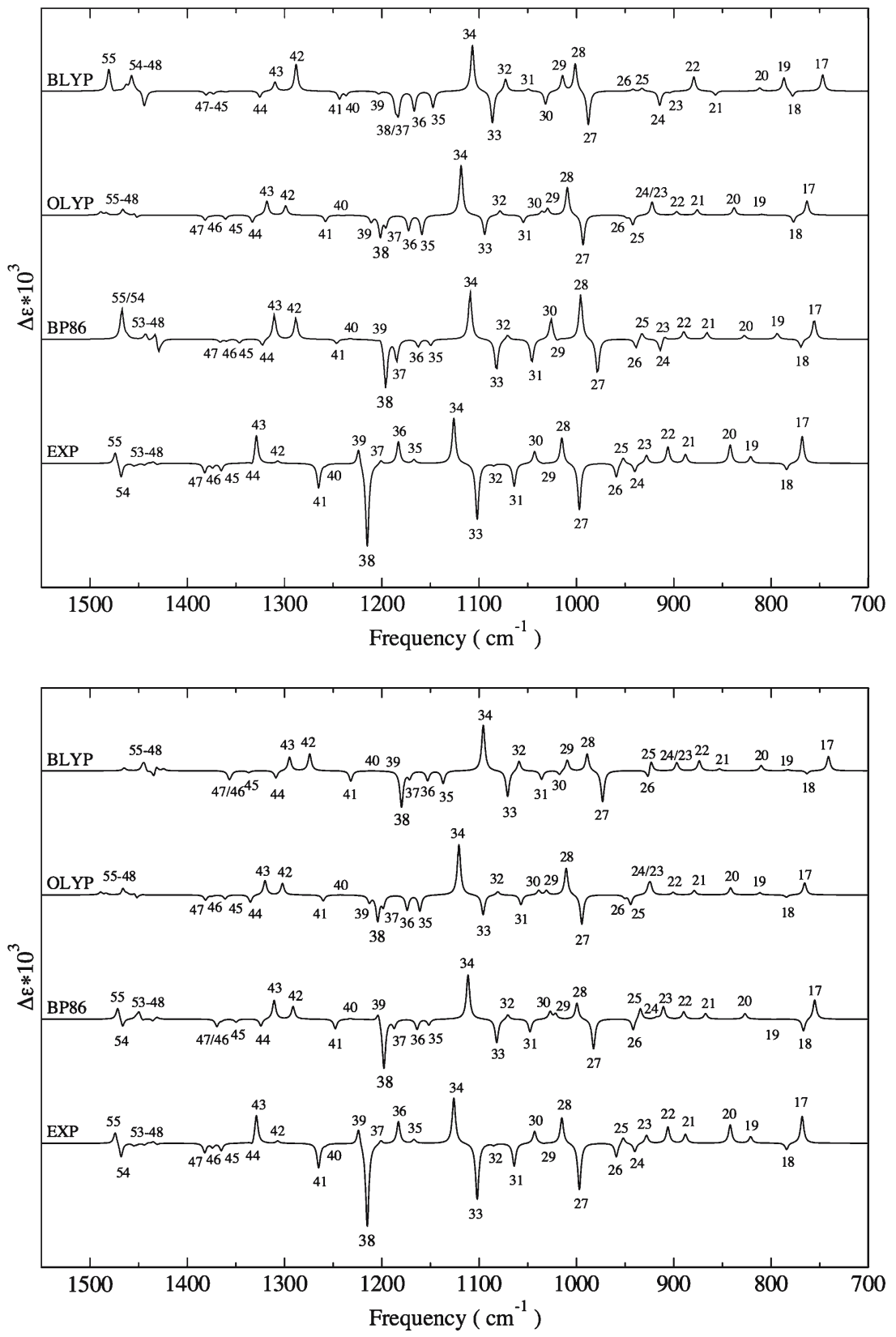

i.e. same sign for most modes with significant rotational strengths, there is a fair correspondence between their VCD spectra. The best agreement with experiment is obtained from the BP86 functional, which enables a very clear assignment of the fundamental modes 17-47, 54 and 55. The OLYP and BLYP spectra have about the same accuracy when compared to experiment, with the OLYP spectrum being slightly better.

In the case of the BP86 functional, the sign of the calculated rotational strengths for some of the fundamentals $(32,35-37)$ differs from the experimental spectrum for both ET-pVQZ and TZP basis sets. However, all of these modes have low VCD intensities. The TZP calculation additionally predicts a wrong sign for the weak rotational strengths of the modes 19, 24 and 29. The fundamentals 48-53 overlap in both calculated and experimental spectra.

In the case of the OLYP functional, the VCD spectra obtained with the TZP and ET-pVQZ basis sets are very similar. The modes $17-47$ can be clearly assigned while the modes 48-55 are unresolved for both basis sets used. Again, the rotational strengths for some of the low-intensity modes (24, 25, 29, 32, 35-37 and 39) have opposite sign compared to the experimental values.

For the BLYP spectra, the basis set effect when changing from TZP to ET-pVQZ is more pronounced (see 
fundamentals $21-26$ and 37-39 in Figs. 2 and 3). The fundamentals $17-47$ can be clearly assigned while fundamentals 48-55 are unresolved for both ET-pVQZ and TZP spectra. The ET-pVQZ predicts the wrong sign for the fundamentals $21,23,26,29-31,35-37$ and 39 . The TZP basis yield the wrong sign for the fundamentals 24 , 29, 30, 35-37, 39, and 40.

To sum up, the BP86 spectra agree very well with the experimental spectrum; the differences between the TZP and ET-pVQZ spectra are very small. The larger ET-pVQZ basis improves the agreement with experiment for the fundamentals 19,24 , and 29 , for which the correct sign is obtained. The OLYP functional yields spectra less accurate than the BP86 functional but better than the BLYP functional when the ET-pVQZ basis sets are used. For TZP basis sets the OLYP and BLYP functionals yield VCD spectra of similar accuracy when compared to the experimental spectrum. Thus, for $\alpha$-pinene, the relative accuracy of the functionals is BP86 $>$ OLYP $\geq$ BLYP.

Since hybrid functionals are very popular for the calculation of VCD spectra, in what follows we compare the VCD spectra obtained here using pure density functionals with the results from calculations employing hybrid functionals that have been published before. In Ref. [33] the IR and VCD spectra of $\mathbf{2}$ have been calculated using the B3PW91 and B3LYP hybrid functionals and TZ2P basis set. The two hybrid functionals yielded very similar spectra that compare well with experiment. The B3PW91 spectra are somewhat better. Figure 4 shows the BP86/TZP, BP86/ET-pVQZ, B3PW91/TZ2P and B3LYP/TZ2P VCD spectra together with the experimental one. For a fair comparison, the B3PW91 and B3LYP spectra in Fig. 4 were obtained by broadening the rotational strengths and frequencies published in Ref. [33] using the same technique used to obtain the BP86 spectra. As can be seen in Fig. 4 all four calculated spectra are qualitatively very similar and lead to an identical assignment of all fundamentals. The modes 17 , $27-28,33-34,38$ and 43 with relative high VCD intensities, that stand out in the experimental spectrum, are reproduced by all calculated spectra. For the modes with less intense VCD signals differences exist. Thus, for modes 21-26 the spectra obtained with BP86 are clearly superior to the ones obtained with hybrid functionals. The BP86/ET-pVQZ is the only spectrum that clearly reproduces the experimental pattern; the BP86/TZP spectrum shows that there is a basis set effect only for mode 24, which has low intensity (positive) in TZP basis. The B3PW91 and B3LYP spectra have been judged to be in qualitative disagreement with the experiment [33]. In the case of modes 35-37 the BP86 predicts the wrong sign for all three modes for both ET-pVQZ and TZP basis sets; the B3PW91 predicts the wrong sign only for mode 37 while B3LYP yields the wrong sign for the modes 35 and 37.

In conclusion, we would argue that the BP86/ETpVQZ yielded overall the best agreement with experiment, even though only marginally so in comparison to B3PW91/TZ2P, and not in every detail. The smaller TZP basis hardly deteriorates the BP86/ET-pVQZ results, and for instance BP86/TZP apparently performs better than B3LYP/TZ2P. One certainly does not obtain superior performance with the more expensive hybrid functionals.

\subsubsection{VCD spectra of Troger's base (3)}

The absolute configuration of $\mathbf{3}$ has been determined by a combination of experiment and calculation [37]. The calculated (BP86/TZP and OLYP/TZP) and experimental VCD spectra are shown in Fig. 5. The experimental data are taken from Ref. [37]. A list of calculated and experimental frequencies and rotational strengths is shown in Table 3 in the supplementary material (see ESM).

As can be seen from Fig. 5 both calculated spectra compare very well with experiment. The OLYP and BP86 functionals yield frequencies that are very close to the experimental ones; there is no need for scaling. The OLYP frequencies are slightly better than the BP86 ones. The calculated rotational strengths are also in very good agreement with the experiment, permitting an unambiguous assignment of almost all fundamentals from 33 to 87 that have a considerable VCD intensity. For the few modes with low VCD intensities or those vibrations with overlapping peaks, a clear assignment was not possible. Thus, both functionals predict the wrong sign for the modes $33,40,46,68,70,71$; BP86 also predicts the incorrect sign for mode 67 . The modes 54-56, 62, 63, 71 and 72 have very low VCD intensities which prevents a detailed assignment for both functionals; the same holds true for the modes 75-78 calculated with OLYP. To sum up, the OLYP and BP86 functionals predict VCD spectra of $\mathbf{3}$ that reproduce the experimental spectrum very well. The frequencies and rotational strengths from OLYP are slightly better than the ones obtained with BP86. We refrain from performing calculations with larger basis sets since the results obtained for $\alpha$-pinene indicate that the spectra are converged with respect to the size of the basis set.

In Ref. [37] the VCD spectra of 3 have been calculated using the B3PW91 and B3LYP hybrid functionals and 6-31G* basis sets. In Fig. 6 we present a comparison between the VCD spectra obtained here with the BP86 and OLYP functionals and TZP basis sets and the 
Fig. 4 VCD spectra of $\alpha$-pinene (2). Comparison between the experimental spectrum and spectra obtained with the OLYP, BP86 (this work), B3PW91 and B3LYP (from Ref. [33]) functionals
Fig. 5 VCD spectra for Troger's Base (3). Comparison of experiment with TZP calculations
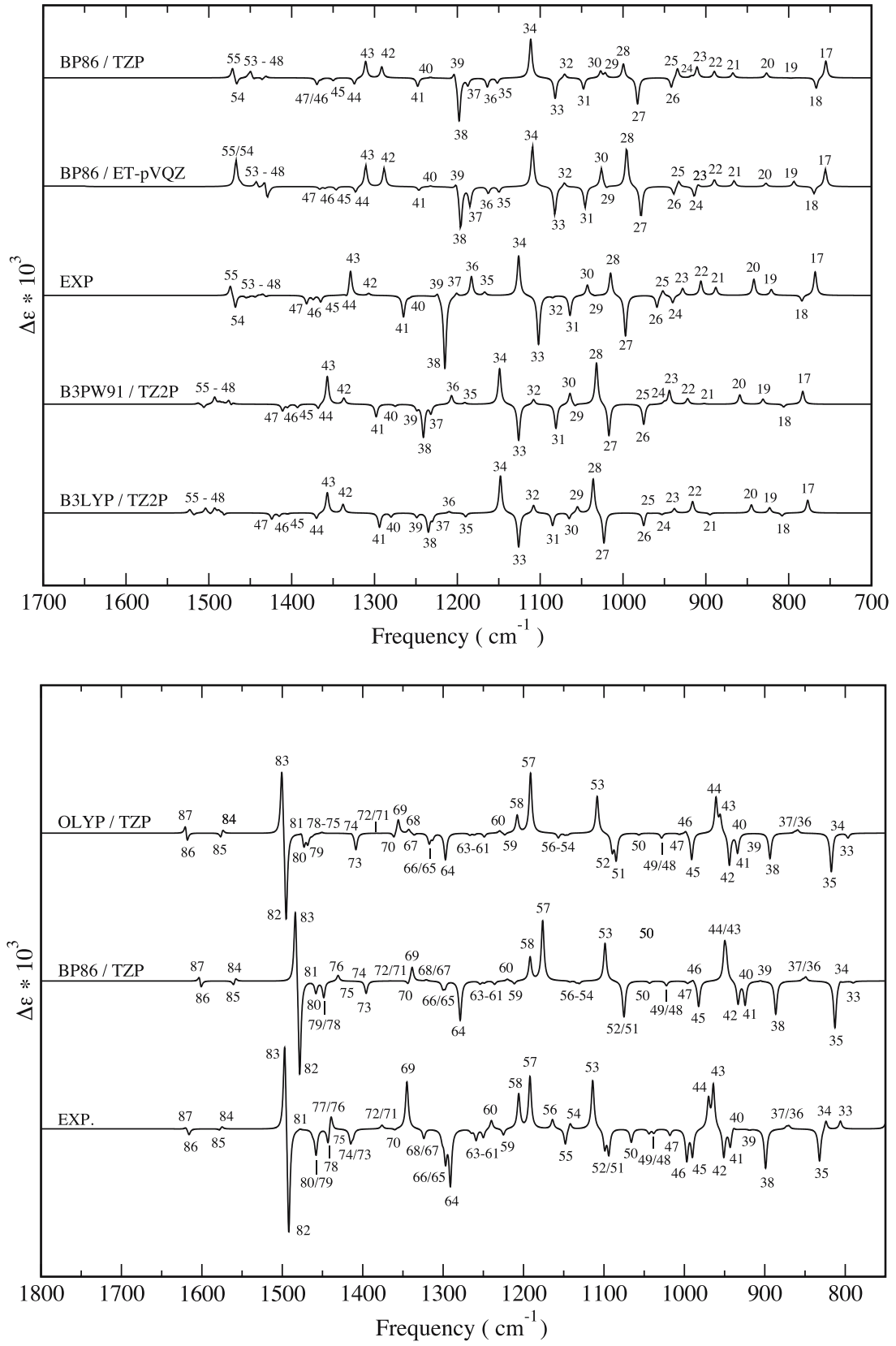

B3PW91/6-31G* spectrum. Due to the similarity of the B3PW91 and B3LYP spectra only the first one was considered here. The B3PW91 spectrum in Fig. 6 was obtained by broadening the rotational strengths and frequencies published in Ref. [37] using the same technique used to obtain the BP86 and OLYP spectra. As can be seen from Fig. 6 the frequencies obtained with the BP86 and OLYP functionals are superior to the ones obtained with B3PW91 when compared to experiment. Apart from the frequencies, the three functionals predict spectra that are qualitatively very similar. All significant features of the experimental spectrum, i.e. modes 35 , $38,42-45,51-53,57,58,64,69$ and 82-83, are repro- duced by all calculated spectra. The OLYP and BP86 spectra are somewhat closer to the experimental spectrum. This is mostly due to their superior frequencies but also because they reproduce better some intensities of the experimental spectrum. Thus, mode 41, is unresolved in the B3PW91 spectrum while it is unambiguously assigned in the BP86 and OLYP spectra. Modes 51 and 52 overlap in the BP86 and B3PW91 spectra while they can be clearly distinguished in the OLYP and experimental spectra.

In conclusion, the OLYP, BP86 and B3PW91 functionals yielded VCD spectra in very good agreement with the experiment that lead to identical assignment 
Fig. 6 VCD spectra of Troger's Base (3).

Comparison between the experimental spectrum and spectra obtained with the OLYP, BP86 (this work), B3PW91 and B3LYP (from Ref. [37]) functionals

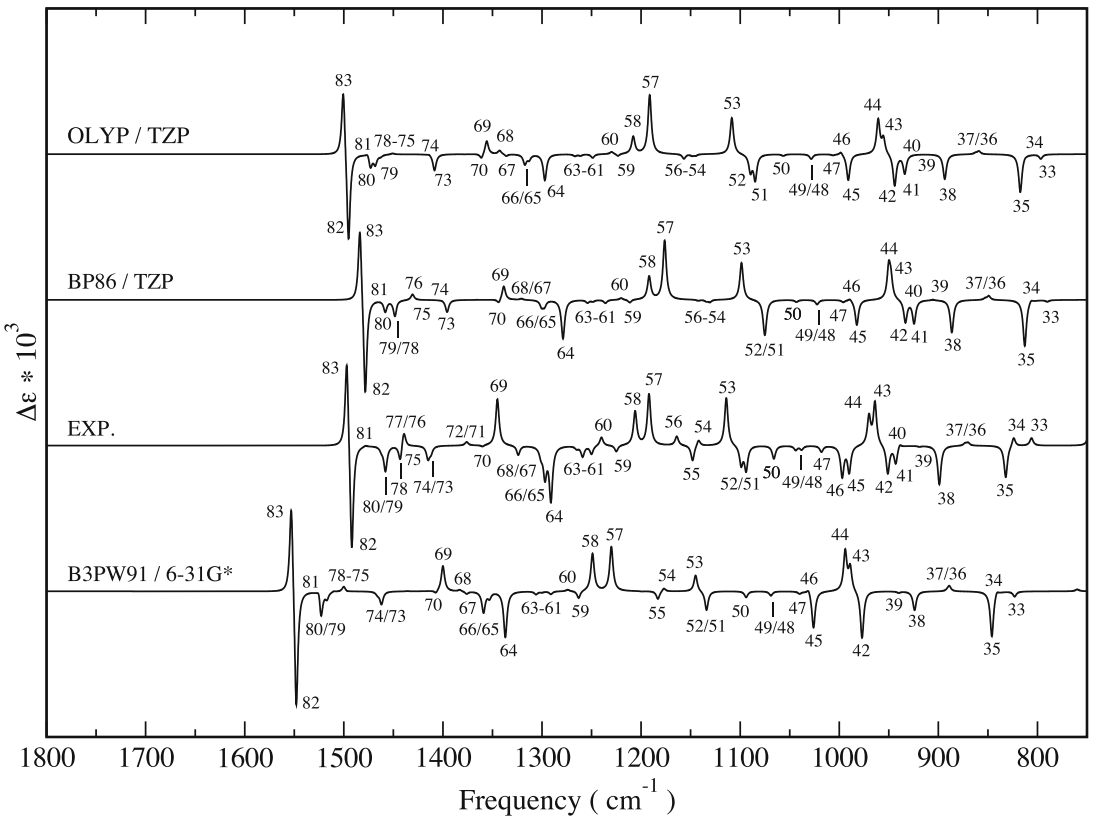

of all the modes with reasonable intensities. The spectra obtained with OLYP and BP86 are better than the B3PW91, mostly due to their superior force fields.

\section{VCD of hexa- (4) and hepta-helicene (5)}

We performed calculations for $\mathbf{4}$ and $\mathbf{5}$ using the OLYP functional and TZP basis sets. The molecules are depicted in Fig. 7. The $C_{2}$-axis was chosen to be the $Z$-axis of the coordinate system. The graphics presented in Figs. 7 and 10 have been created using the program XCrySDen [38]. The frequencies and rotational strengths for $\mathbf{4}$ and $\mathbf{5}$ are shown in Tables 4 and 5 in the supplementary material (see ESM). The VCD spectra are presented in Fig. 8. Only the range from 900 to $1650 \mathrm{~cm}^{-1}$ of the VCD spectra is shown since this range is significant for the present study.
An experimental spectrum of $\mathbf{5}$ has been published in Ref. [10], together with a spectrum calculated with B3LYP $[39,40]$ and a 6-31G(d,p) basis. This small basis set is of double- $\zeta$ quality, augmented with polarization functions, so that the results would probably change a bit when larger basis sets are employed. Furthermore, an empirical scaling factor had to be applied to the vibrational frequencies. Nevertheless, that calculation reproduced many of the features observed in experiment, and thus perfectly served the purpose to assign the absolute configuration of the hepta-helicene enantiomer under study. Our calculation reproduces the significant features of the experimental VCD spectrum: the combination of a positive and a negative peak at $950 \mathrm{~cm}^{-1}$ can be observed, as well as a series of negative peaks between 1100 and $1300 \mathrm{~cm}^{-1}$. There are three pronounced negative peaks in the experimental spectrum at approximately 1470,1510 , and $1610 \mathrm{~cm}^{-1}$, which are well reproduced in our simulation by the peaks 108 ,
Fig. 7 Equilibrium geometries (OLYP/TZP) for hexa-helicene (4) and hepta-helicene (5)

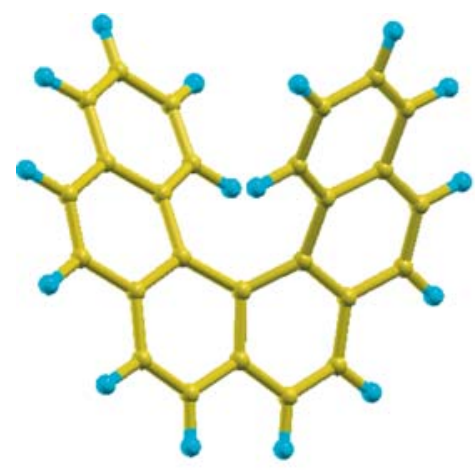

(4)

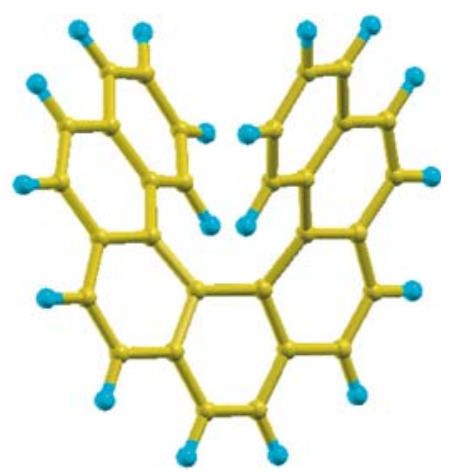

(5) 
Fig. 8 VCD spectra of hexaand hepta-helicene (OLYP/TZP)

Fig. 9 VCD spectra of hexaand hepta-helicene (OLYP/TZP)
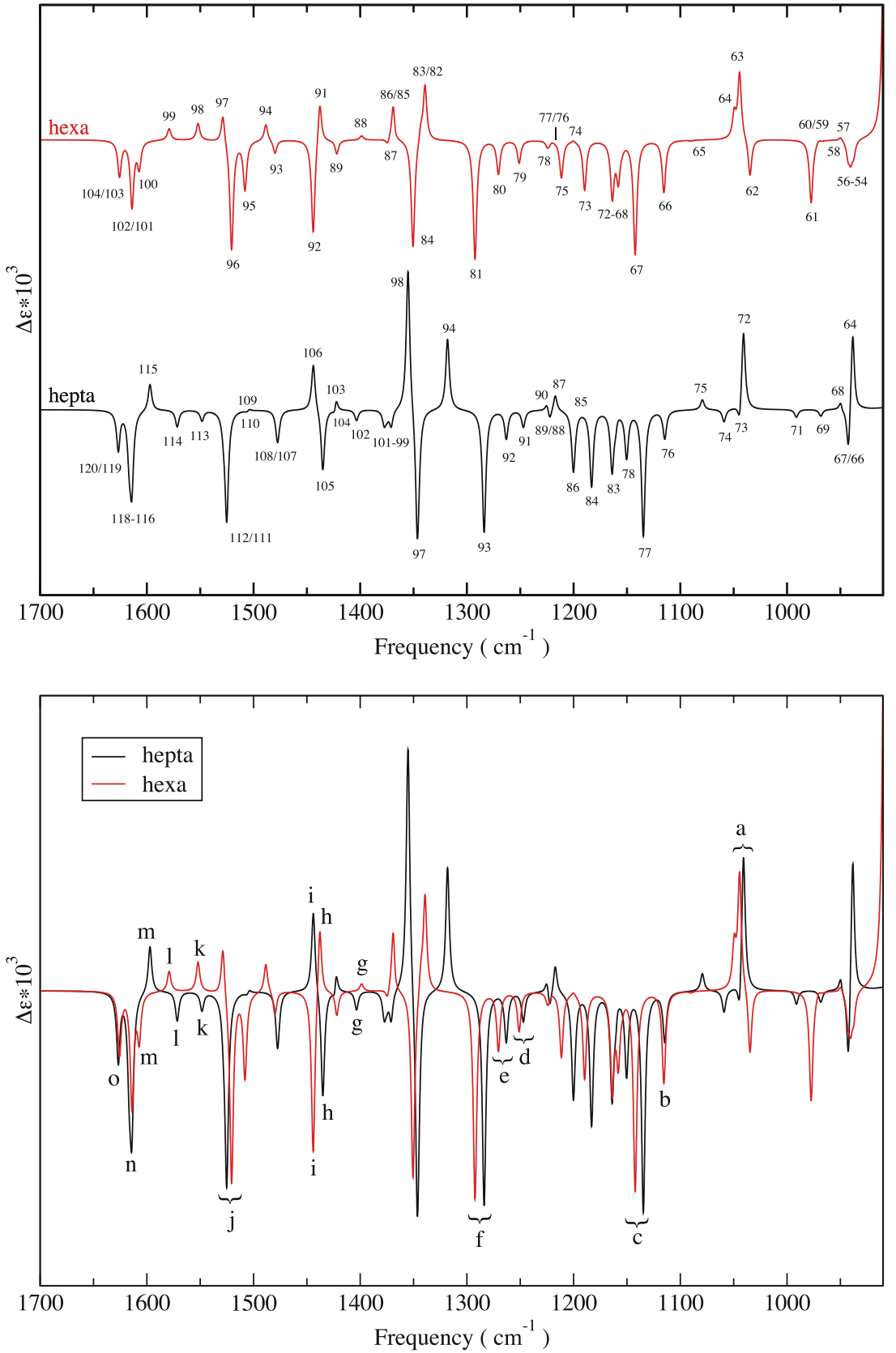

111 , and the overlap of the modes 116-120. We also note that there is a good general agreement between our OLYP/TZP calculation and the B3LYP/6-31G(d,p) calculation in Ref. [10].

As can be seen from Fig. 8 there are many modes with comparable frequencies and similar (absolute) rotational strengths in the two molecules, such as modes 76-86, 91-93, 94-98, and 116 to 120 of hepta-helicene and the modes $66-75,79-81,82-86$, and $100-104$ of hexa-helicene, respectively. Plotting the spectra on top of each other (see Fig. 9) clearly exhibits the pairs of similar modes. We can distinguish pairs for which the two modes have about the same frequency and the same sign for the rotational strength and pairs that have about the same frequency but different sign for the rotational strengths. We have labeled relevant pairs with $a$ to $o$, where we have verified that the vibrational motions in the modes of a pair are also physically similar (see below in what sense precisely).

The rotational strengths, frequencies and the symmetry species of the labeled modes of both molecules are shown in Table 3. By analyzing the data in Table 3 three observations can be made. First, when the modes in a pair have the same symmetry, either both $A$ or both 
Fig. 10 The pair of normal modes labeled $i$ in Fig. 9. a Hexa-helicene, mode of irrep B; b hepta-helicene, mode of irrep A

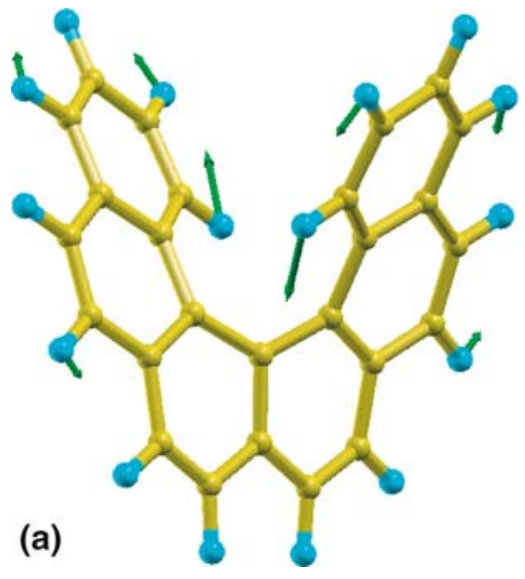

$B$, they have the same sign of the rotational strength. Second, when the modes of the pair have different symmetries, we invariably find different signs of the rotational strengths. Third, we observe that the absolute magnitudes of the rotational strengths of the modes of a pair seem to be roughly correlated. If the rotational strength is large for one of the molecules it is also large for the other. This correspondence is by no means quantitative, the difference in rotational strengths may amount to $50 \%$.

The correlation between the spectra of the hexa- and hepta-helicenes as displayed in Fig. 9 is sufficiently striking that we feel it calls for an explanation. We will therefore consider this case in some more detail. We note that the $C_{2}$ symmetry of these molecules is very common in chiral molecules, so this analysis may find wider application.

As it is clear from Fig. 7 the $C_{2}$-axis divides the molecules in two symmetry related wings (referred as I and II). The benzene rings at the outer ends of the molecules show a certain similarity in hexa-and hepta-helicene. The wings with these similar rings are connected by the central ring(s). Looking at how the nuclei are displaced in the normal modes of the pairs of modes we have singled out (labeled with $a$ to $o$ in Fig. 9), we find that the nuclei are displaced in an almost identical way in the two modes (i.e. in the two molecules). As an example, in Fig. 10 the nuclear displacements for the pair of normal modes labeled $i$ in Fig. 9 are shown. As can be seen, even though the amplitudes of the displacements are a bit different in the two molecules, the nuclei execute essentially the same type of movements, except that of course in the B mode the atoms of the two wings move in opposite directions. This makes the similar frequencies understandable. It is not always true that the atoms of the connecting rings practically do not move, as in the $i$ pair, but then the similarity of the motions of the wings is still sufficiently dominant to provide similar frequencies. We are not dealing with quantitative equalities anyway, but with qualitative similarities. The similar vibrational motions are expected to lead to certain relationships in the electric and magnetic dipole transition moments. To see how this can be understood, we consider a pair of modes. For simplicity, we consider A and $\mathrm{B}$ modes belonging to the same helicene; the conclusions will be applicable to our pairs of modes in hexaand hepta-helicene, since we use the similarity in the vibrations of these systems, cf. Fig. 10.

The Cartesian components of the total electric and magnetic dipole transition moments are given by Eqs. (2) and (3). For brevity in what follows a general transition dipole moment-when we do not specialize to either electric or magnetic transition dipole-will be referred to as just $\boldsymbol{\mu}$ and will be written as:

$\mu_{\beta}=\left\langle 0\left|\hat{\mu}_{\beta}\right| 1\right\rangle=\sum_{\lambda \alpha} T_{\alpha \beta}^{\lambda} S_{\lambda \alpha, i}$

where $\mu_{\beta}$ denotes the $\beta$ Cartesian component of the total electric or magnetic dipole transition moment while $T_{\alpha \beta}^{\lambda}$ denotes $P_{\alpha \beta}^{\lambda}$ or $M_{\alpha \beta}^{\lambda}$. When a specific dipole transition moment is referred to, its appropriate notation $\left(\boldsymbol{\mu}_{E}\right.$ or $\left.\boldsymbol{\mu}_{M}\right)$ will be used. Note that the contribution to $\mu_{\beta}$ from a given nucleus $\lambda$ is given by:

$\mu_{\beta}^{\lambda}=\sum_{\alpha} T_{\alpha \beta}^{\lambda} S_{\lambda \alpha, i}$

Further on, the transition dipole moments are written as the sum of contributions from the two wings (I and II):

$\mu_{\beta}=\mu_{\beta}^{I}+\mu_{\beta}^{I I}=\sum_{\lambda \alpha} T_{\alpha \beta}^{\lambda} S_{\lambda \alpha, i}+\sum_{\lambda^{\prime} \alpha} T_{\alpha \beta}^{\lambda^{\prime}} S_{\lambda^{\prime} \alpha, i}$

where

$\mu_{\beta}^{I}=\sum_{\lambda \alpha} T_{\alpha \beta}^{\lambda} S_{\lambda \alpha, i} \quad$ and $\quad \mu_{\beta}^{I I}=\sum_{\lambda^{\prime} \alpha} T_{\alpha \beta}^{\lambda^{\prime}} S_{\lambda^{\prime} \alpha, i}$ 
In Eqs. (37) and (38) $\lambda$ runs over one half of the nuclei (those of wing I) while $\lambda^{\prime}$ runs over the other half of the nuclei (those of wing II). Thus, $\boldsymbol{\mu}^{I}$ and $\boldsymbol{\mu}^{I I}$ are the contributions of each wing to the total dipole transition moments.

In the case of $C_{2}$ symmetry, the normal modes $\left(\mathbf{S}_{\lambda \alpha, i}\right)$ belong to either the symmetric (A) or the antisymmetric (B) irreducible representation. Thus, for a pair of atoms that are related by symmetry $\left(\lambda\right.$ and $\left.\lambda^{\prime}\right)$ the normal modes satisfy the following relations:

$S_{\lambda^{\prime} \alpha, i}=\left\{\begin{array}{rr}-S_{\lambda \alpha, i} & \text { for } \alpha=x \\ -S_{\lambda \alpha, i} & \text { for } \alpha=y \\ S_{\lambda \alpha, i} & \text { for } \alpha=z\end{array}\right.$

for a mode of irrep A, and

$S_{\lambda^{\prime} \alpha, i}= \begin{cases}S_{\lambda \alpha, i} & \text { for } \alpha=x \\ S_{\lambda \alpha, i} & \text { for } \alpha=y \\ -S_{\lambda \alpha, i} & \text { for } \alpha=z\end{cases}$

for a normal mode of irrep B. We will be considering the idealized case of an A mode and a B mode which have identical nuclear displacements $\mathbf{S}_{\lambda \alpha, i}$, and $\mathbf{S}_{\lambda^{\prime} \alpha, i}$ for the symmetry related atoms according to Eqs. (39) and (40).

The transformation properties of the tensors $M_{\alpha \beta}^{\lambda}$ and $E_{\alpha \beta}^{\lambda}$, in the case of $C_{2}$ symmetry are:

$T_{\alpha \beta}^{\lambda^{\prime}}= \begin{cases}T_{\alpha \beta}^{\lambda} & \text { for } \alpha=\beta \\ T_{\alpha \beta}^{\lambda} & \text { for } \alpha \neq \beta ; \alpha, \beta=x, y \\ -T_{\alpha \beta}^{\lambda} & \text { for } \alpha \neq \beta ; \alpha=z, \beta=x, y \\ -T_{\alpha \beta}^{\lambda} & \text { for } \alpha \neq \beta ; \alpha=x, y, \beta=z\end{cases}$

where $\lambda$ and $\lambda^{\prime}$ are two symmetry related atoms:

By inserting Eqs. (39), (40) and (41) into (36), the relations between the Cartesian components of $\mu^{\lambda}$ and $\boldsymbol{\mu}^{\lambda^{\prime}}$ are obtained:

$\mu_{x}^{\lambda^{\prime}}=-\mu_{x}^{\lambda} ; \mu_{y}^{\lambda^{\prime}}=-\mu_{y}^{\lambda} ; \mu_{z}^{\lambda^{\prime}}=\mu_{z}^{\lambda}$

for a mode of irrep A, and

$\mu_{x}^{\lambda^{\prime}}=\mu_{x}^{\lambda} ; \quad \mu_{y}^{\lambda^{\prime}}=\mu_{y}^{\lambda} ; \mu_{z}^{\lambda^{\prime}}=-\mu_{z}^{\lambda}$

for a mode of irrep B.

Obviously, in an A mode the $\mathrm{x}$ - and y-components of a $\boldsymbol{\mu}^{\lambda}$ will be canceled by those of $\boldsymbol{\mu}^{\lambda^{\prime}}$, so both the electric and magnetic transition dipoles will be directed along the $Z$-axis (the $C_{2}$-axis). For a $\mathrm{B}$ mode the cancelation occurs for the z-component, so in that case the transition dipoles are perpendicular to the $Z$-axis. We note that when we compare our idealized A and B modes, which will have the same $\mu^{\lambda}$ 's (in wing I), the consequence of Eqs. (42) and (43) is that for the B mode the $\boldsymbol{\mu}^{\lambda^{\prime}}$ s in wing II change sign compared to the $\boldsymbol{\mu}^{\lambda} \mathrm{s}$ of the A mode.

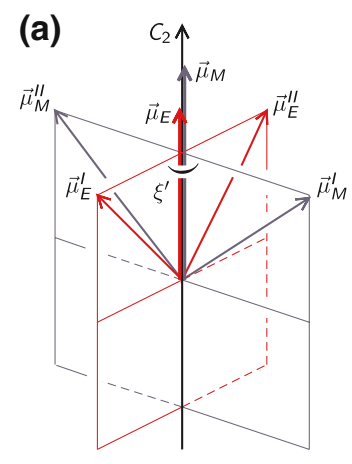

(b)

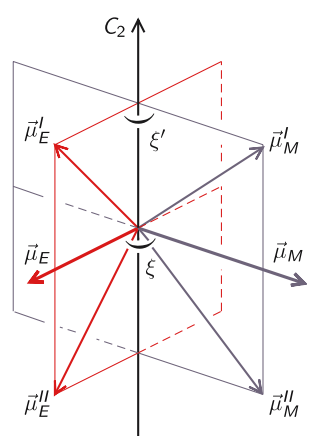

Fig. 11 Electric and magnetic dipole transition moments of the two wings of helicene: a mode of irrep A with parallel $\mu_{E}$ and $\mu_{M}$; b mode of irrep B
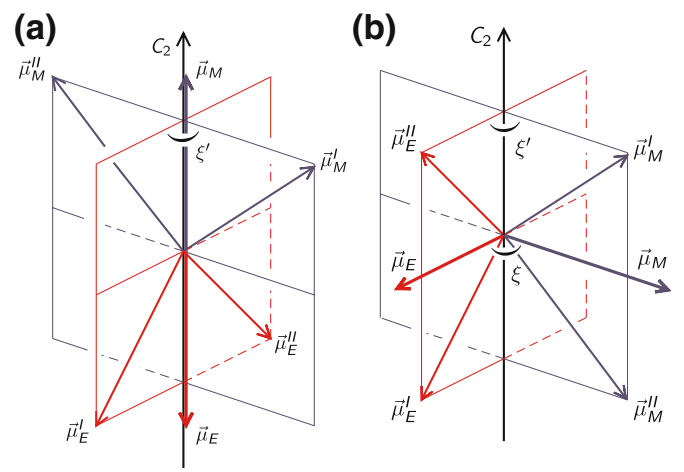

Fig. 12 Electric and magnetic dipole transition moments of the two wings of helicene: a mode of irrep A with anti-parallel $\mu_{E}$ and $\boldsymbol{\mu}_{M} ; \mathbf{b}$ mode of irrep B

Let us first make an observation on our pairs with modes of the same symmetry (A for the pairs $c, e, n$ and $\mathrm{B}$ for $a, b, d, f, j, o)$. We have verified that these modes in the two helicenes approach the situation that there are similar displacements for the atoms in the wings of heptahelicene as in the wings of hexa-helicene, and that there are similar APTs and AATs on those atoms. Then straightforwardly the conclusion follows that the total $\mu_{E}$ and $\mu_{M}$ will be similar, and therefore also the rotational strengths. Turning to the pairs of modes of different symmetry, we can use the Eqs. (42) and (43) to deduce the various possibilities. Since the rotational strength is defined as the imaginary part of the scalar product between the electric and magnetic dipole transition moments, Eq. (1), its sign is determined by $\cos \xi$ where $\xi$ is the angle between the total electric and magnetic dipole transition moments. Thus, for $\xi<90^{\circ}$ the rotational strength is positive while for $90^{\circ}<\xi<180^{\circ}$ it is negative. For a totally symmetric mode $\boldsymbol{\mu}_{E}$ and $\boldsymbol{\mu}_{M}$ can be either parallel $\left(\xi=0^{\circ}\right)$ or anti-parallel $\left(\xi=180^{\circ}\right)$. Therefore, the rotational strength is positive in the first situation and negative in the second one. We will treat 
Table 3 Frequencies, rotational strengths and the angle $\xi^{\prime}$ of modelecules $\mathbf{4}$ and $\mathbf{5}$ for the pairs of normal modes labeled in Fig. 7

\begin{tabular}{|c|c|c|c|c|c|c|c|c|}
\hline \multirow[b]{2}{*}{ Pairs } & \multicolumn{4}{|c|}{ Hexa-helicene } & \multicolumn{4}{|c|}{ Hepta-helicene } \\
\hline & Freq. & Sym. & $\xi^{\prime}$ & Rot. Str. & Freq. & Sym. & $\xi^{\prime}$ & Rot. Str. \\
\hline$a$ & 1044 & B & 53.5 & 12.9 & 1041 & B & 52.9 & 15.8 \\
\hline$b$ & 1115 & B & 106.7 & -9.4 & 1114 & $\mathrm{~B}$ & 106.1 & -5.1 \\
\hline$c$ & 1142 & A & 113.6 & -19.8 & 1134 & A & 147.4 & -22.4 \\
\hline$d$ & 1251 & B & 102.8 & -3.7 & 1247 & B & 101.0 & -2.7 \\
\hline$e$ & 1270 & A & 162.4 & -5.4 & 1263 & A & 92.4 & -4.5 \\
\hline$f$ & 1292 & $\mathrm{~B}$ & 137.1 & -18.8 & 1284 & $\mathrm{~B}$ & 170.9 & -19.3 \\
\hline$g$ & 1399 & A & 93.4 & 0.5 & 1403 & $\mathrm{~B}$ & 111.1 & -1.5 \\
\hline h & 1438 & A & 176.6 & 6.0 & 1435 & B & 172.1 & -8.7 \\
\hline$i$ & 1444 & $\mathrm{~B}$ & 153.2 & -13.5 & 1444 & A & 177.0 & 6.6 \\
\hline$j$ & 1520 & B & 169.9 & -15.0 & 1525 & B & 175.0 & -14.3 \\
\hline$k$ & 1552 & A & 165.5 & 2.1 & 1548 & $\mathrm{~B}$ & 119.4 & -1.3 \\
\hline$l$ & 1579 & $\mathrm{~B}$ & 77.8 & 1.3 & 1571 & A & 10.1 & -2.1 \\
\hline$m$ & 1607 & $\mathrm{~A}$ & 99.5 & -3.2 & 1597 & $\mathrm{~B}$ & 61.2 & 3.4 \\
\hline$n$ & 1614 & A & 146.4 & -2.7 & 1614 & A & 152.0 & -4.8 \\
\hline$o$ & 1625 & B & 106.4 & -1.8 & 1626 & B & 115.0 & -5.2 \\
\hline
\end{tabular}

Frequencies are given in $\mathrm{cm}^{-1}$, rotational strengths are given in $10^{-44} \mathrm{esu}^{2} \mathrm{~cm}^{2}, \xi^{\prime}$ is given in degrees

these two cases separately. In Fig. 11a the case A-positive and Fig. 12a the case A-negative are presented. In these figures we geometrically demonstrate what the situation can be for the B counterpart in the pair of modes. We consider in Fig. 11a how the total electric and magnetic transition dipoles are built up from the contributions of wings I and II, where the latter obey Eq. (42), which leads to the "mirroring" of $\boldsymbol{\mu}^{I}$ with respect to the $C_{2}$-axis to obtain $\mu^{I I}$. The orientation of the plane containing $\boldsymbol{\mu}_{E}^{I}$ and $\boldsymbol{\mu}_{E}^{I I}$ relative to the plane of $\boldsymbol{\mu}_{M}^{I}$ and $\boldsymbol{\mu}_{M}^{I I}$ is not known beforehand. We denote the angle between these two planes by $\xi^{\prime}$, and note that $\xi^{\prime}$ does not play a role in determining the rotational strength in the A mode, since the total $\mu_{E}$ and $\mu_{M}$ are along the $Z$-axis. When going to the B mode, $\mu_{E}^{I}$ and $\mu_{M}^{I}$ remain unchanged while $\boldsymbol{\mu}_{E}^{I I}$ and $\boldsymbol{\mu}_{M}^{I I}$ change sign, see Fig. 11b. The angle $\xi^{\prime}$ now becomes the angle between the total $\mu_{E}$ and $\mu_{M}$, i.e. is equal to $\xi$, and $\cos \xi^{\prime}$ is now determining the sign and magnitude of the rotational strength. Since there is no a priori restriction for $\xi^{\prime}$, the rotational strength can be positive or negative depending on the value of $\xi^{\prime}$.

In Fig. 12b we show the construction of $\mu_{E}$ and $\mu_{M}$ of the $\mathrm{B}$ mode when the A mode has negative rotational strength. Again the angle $\xi^{\prime}$ determines the result for the B mode, and cannot be related straightforwardly with the (sign of) the oscillator strength of the corresponding A mode. We have calculated the $\xi^{\prime}$ angles for all the modes in the pairs we are considering; they are given in Table 3. We note in passing that for the pairs with modes of the same symmetry, the $\xi^{\prime}$ angles in the hexa- and hepta-helicene are indeed in reasonable agreement. This substantiates our finding that in these cases we are really dealing with modes in the two molecules that strongly resemble each other. For the pairs with modes of different symmetry ( $g, h, i, k$ and $l$ ) the correspondence between the $\xi^{\prime}$ angles is less good, indicating that the correspondence in the vibrational motions is less clear than in the case of modes which have the same symmetry. Still, there is qualitative agreement. If we consider the $i$ pair, we have $\xi^{\prime}$ values of $26.8^{\circ}$ and $3.0^{\circ}$, which have cosine values of 0.89 and 0.99 , i.e only $10 \%$ different.

We conclude that we have been able to understand the occurrence of pairs of modes with similar frequency in hexa- and hepta-helicene, which when they have the same symmetry also lead to rotational strengths of the same sign and roughly similar magnitude. We have not been able to fully rationalize our observation that in the pairs with modes of different symmetry the rotational strengths must have different sign. There are more factors that determine the sign and magnitude of the rotational strength of the $\mathrm{B}$ mode compared to the A mode, such as differences in the geometries of the two molecules, the angle which the $\boldsymbol{\mu}_{M}^{I}$ and $\boldsymbol{\mu}_{E}^{I}$ vectors make with the $Z$-axis, and their absolute magnitudes, which we cannot take into account in our simple model.

Nevertheless, we demonstrated-using the geometrical relations between contributions of the transition dipole moments from the two wings of the moleculesthat the rotational strengths of corresponding normal modes in compounds A and B can have different sign.

\section{Conclusions}

The comparison of AATs calculated with the distributed origin gauge and the common origin gauge has proven that the LAO-STO-based implementation works 
correctly, as the two origin gauges yield virtually identical AATs and therefore origin independent rotational strengths. Thus, the calculations are not sensitive to the special techniques which have to be used in connection with STOs, e.g., the numerical integration for the matrix elements. It was demonstrated that the sum-rules are fulfilled and quite accurate results can be obtained for the AATs even with moderately large STO basis sets like TZP. In these cases, the sum rules are fulfilled to within $3 \%$ or less. For the largest basis sets used here, the error in the sum-rules drops to less than $0.7 \%$. Although the AATs obtained with a TZP basis are apparently almost converged w.r.t. the basis set size, we would like to note that calculations with the much larger ET-pVQZ basis set are straightforward for reasonably large molecules with our efficient implementation, which heavily makes use of the parallelization and linear-scaling techniques available in ADF.

The study of different combinations of functionals and basis sets for VCD spectra underlines the importance of accurate vibrational frequencies and the corresponding normal modes. Therefore, the VCD spectra from BP86 calculations are usually superior to those from BLYP calculations in terms of agreement with experimental spectra. This is due to the well-known feature of BP86 to yield harmonic force fields in close agreement with experiment [34-36]. VCD spectra from OLYP calculations are comparable to or even slightly better than those from BP86. This is because the two functionals yield similar force fields. Regarding the basis set dependence of the VCD spectra it turns out that a TZP basis set is sufficiently large for calculations of reasonable accuracy. Our tests, performed on many chiral molecules suggested that BP86/TZP is always a safe choice for a good agreement with experiment.

Although the most popular exchange-correlation functionals for VCD calculations are the B3PW91 and B3LYP hybrid functionals, calculations with the nonhybrid functionals employed here, i.e., BP86 and OLYP, have yielded improved frequencies and rotational strengths that are at least as good. Another advantage of these functionals is that efficient and accurate densityfitting techniques can readily be applied in order to reduce the computational cost.

In our final investigation of the VCD spectra of hexaand hepta-helicene, we have shown that it is possible to find relationships between the rotational strengths of corresponding vibrations of the two molecules which are of similar character, i.e., which involve similar motions of (groups of) atoms. It was shown that-based on the irreps the normal modes belong to-the rotational strengths either keep or change sign. This leads to parts of the spectra that are very similar for both molecules, while other parts more or less fulfill mirror-image conditions.

We have thus demonstrated that our implementation is correct and therefore can be applied for theoretical predictions of VCD spectra, which, as already shown $[32,33,37,41-45]$, is an important pre-requisite for interpretations of complicated experimental spectra of larger molecules, the assignment of absolute configurations of chiral substances, and the detection of specific interactions in chiral super-molecular aggregates, which manifest themselves in molecular vibrations.

Acknowledgements We acknowledge the stimulus from discussion and interest of Prof. Jochen Autschbach and Brandon C. Mort of University of New York at Buffalo. J. N. acknowledges funding by a Liebig-Stipendium of the Fonds der Chemischen Industrie (FCI).

Appendix 1: First-order density from a magnetic-field perturbation

The first order perturbation Hamiltonian, $H^{(1)}$, for a magnetic field perturbation is purely complex [19]:

$h^{(1), H}=-i \mathbf{B}\left(\frac{\mathbf{r}}{2} \times \nabla\right)$

Thus, from standard perturbation theory the first order perturbed MOs, $\varphi^{(1)}$, are purely complex too. Since the unperturbed MOs, $\varphi_{i}$, are real, the first order density $\rho^{(1)}$ becomes:

$$
\begin{aligned}
\rho^{(1)} & =\sum_{i}^{\mathrm{occ}}\left(\varphi_{i}^{*} \varphi_{i}^{(1)}+\varphi_{i} \varphi_{i}^{(1)^{*}}\right) \\
& =\sum_{i}^{\mathrm{occ}}\left(\varphi_{i} \varphi_{i}^{(1)}-\varphi_{i} \varphi_{i}^{(1)}\right)=0
\end{aligned}
$$

Appendix 2: Contributions from the occ-occ blocks of the $\mathbf{U}^{(1)}$ matrices

In the case of a magnetic-field perturbation the first order perturbed Hamiltonian and MOs are purely complex. Thus, the first order perturbed Fock and overlap matrix elements are purely complex too and fulfill the following relations:

$F_{i j}^{(1), H_{\beta}}=-F_{j i}^{(1), H_{\beta}}, \quad S_{i j}^{(1), H_{\beta}}=-S_{j i}^{(1), H_{\beta}}$

The matrix elements $U_{i j}^{(1), H_{\beta}}$ are purely complex.

When the perturbation is a nuclear displacement, the first order perturbed Hamiltonian and MOs are real, so that: 
$F_{i j}^{(1), R_{\lambda \alpha}}=F_{j i}^{(1), R_{\lambda \alpha}}, \quad S_{i j}^{(1), R_{\lambda \alpha}}=S_{j i}^{(1), R_{\lambda \alpha}}$

The matrix elements $U_{i j}^{(1), R_{\lambda \alpha}}$ are real.

To obtain Eqs. (46) and (47) we have used that the perturbed Hamiltonians are Hermitian and that the unperturbed MOs are real.

As can be seen, the last three terms in Eq. (25) depend on the $\mathbf{U}^{(1)}$ matrices including their occ-occ blocks. The components of the second and third terms in Eq. (25) due to a pair of off-diagonal occ-occ MOs $i$ and $j$ are:

$$
\begin{aligned}
& U_{i j}^{(1), H_{\beta}} \sum_{a, b=1}^{N_{\text {bas }}} C_{a j} C_{b i}\left\langle\chi_{a}^{(1), R_{\lambda \alpha}} \mid \chi_{b}\right\rangle \\
& +U_{i j}^{(1), R_{\lambda \alpha}} \sum_{a, b=1}^{N_{\text {bas }}} C_{a i} C_{b j}\left\langle\chi_{a} \mid \chi_{b}^{(1), H_{\beta}}\right\rangle \\
& +U_{j i}^{(1), H_{\beta}} \sum_{a, b=1}^{N_{\text {bas }}} C_{a i} C_{b j}\left\langle\chi_{a}^{(1), R_{\lambda \alpha}} \mid \chi_{b}\right\rangle \\
& +U_{j i}^{(1), R_{\lambda \alpha}} \sum_{a, b=1}^{N_{\text {bas }}} C_{a j} C_{b i}\left\langle\chi_{a} \mid \chi_{b}^{(1), H_{\beta}}\right\rangle
\end{aligned}
$$

For nuclear displacement perturbation the basis sets are of STO type and therefore $\chi_{a, b}^{(1), R_{\lambda \alpha}}$ and $\chi_{a, b}$ are both real, thus:

$$
\left\langle\chi_{a}^{(1), R_{\lambda \alpha}} \mid \chi_{b}\right\rangle=\left\langle\chi_{b} \mid \chi_{a}^{(1), R_{\lambda \alpha}}\right\rangle
$$

For magnetic field perturbation the basis sets are of LAO type. From Eq. (26) the derivative of an LAO with respect to a magnetic field $\left(\chi_{a, b}^{(1), H_{\beta}}\right)$ is given by:

$\frac{\partial \sigma_{a}^{A}}{\partial H_{\beta}}=\chi_{a}^{(1), H_{\beta}}=-i \frac{e}{\hbar c}\left(r \times R_{A}\right) \chi_{a}$

Therefore, the $\chi_{a, b}^{(1), H_{\beta}}$ are purely complex. Since the $\chi_{a, b}$ are real we have:

$$
\left\langle\chi_{b}^{(1), H_{\beta}} \mid \chi_{a}\right\rangle=-\left\langle\chi_{a} \mid \chi_{b}^{(1), H_{\beta}}\right\rangle
$$

By interchanging the summation indices $a$ and $b$ in the third and fourth terms of Eq. (48) and using Eqs. (49) and (51), Eq. (48) becomes:

$$
\begin{aligned}
& U_{i j}^{(1), H_{\beta}} \sum_{a, b=1}^{N_{\text {bas }}} C_{a j} C_{b i}\left\langle\chi_{a}^{(1), R_{\lambda \alpha}} \mid \chi_{b}\right\rangle \\
& +U_{i j}^{(1), R_{\lambda \alpha}} \sum_{a, b=1}^{N_{\text {bas }}} C_{a i} C_{b j}\left\langle\chi_{a} \mid \chi_{b}^{(1), H_{\beta}}\right\rangle \\
& +U_{j i}^{(1), H_{\beta}} \sum_{a, b=1}^{N_{\text {bas }}} C_{a j} C_{b i}\left\langle\chi_{a} \mid \chi_{b}^{(1), R_{\lambda \alpha}}\right\rangle \\
& -U_{j i}^{(1), R_{\lambda \alpha}} \sum_{a, b=1}^{N_{\text {bas }}} C_{a i} C_{b j}\left\langle\chi_{a}^{(1), H_{\beta}} \mid \chi_{b}\right\rangle
\end{aligned}
$$

Further on, we express the $U_{i j}^{(1), H_{\beta}}$ and $U_{i j}^{(1), R_{\lambda \alpha}}$ in Eq. (52) using Eq. (22)

$$
\begin{aligned}
& \left(-U_{j i}^{(1), H_{\beta}{ }^{*}}-S_{i j}^{(1), H_{\beta}}\right) \sum_{a, b=1}^{N_{\text {bas }}} C_{a j} C_{b i}\left\langle\chi_{a}^{(1), R_{\lambda \alpha}} \mid \chi_{b}\right\rangle \\
& +\left(-U_{j i}^{(1), R_{\lambda \alpha}{ }^{*}}-S_{i j}^{(1), R_{\lambda \alpha}}\right) \sum_{a, b=1}^{N_{\text {bas }}} C_{a i} C_{b j}\left\langle\chi_{a} \mid \chi_{b}^{(1), H_{\beta}}\right\rangle \\
& +U_{j i}^{(1), H_{\beta}} \sum_{a, b=1}^{N_{\text {bas }}} C_{a j} C_{b i}\left\langle\chi_{a} \mid \chi_{b}^{(1), R_{\lambda \alpha}}\right\rangle \\
& -U_{j i}^{(1), R_{\lambda \alpha}} \sum_{a, b=1}^{N_{\text {bas }}} C_{a i} C_{b j}\left\langle\chi_{a}^{(1), H_{\beta}} \mid \chi_{b}\right\rangle
\end{aligned}
$$

Grouping together the terms that have the same $U^{(1)}$ matrix elements, we obtain:

$$
\begin{aligned}
& -U_{j i}^{(1), H_{\beta}} \sum_{a, b=1}^{N_{\text {bas }}} C_{a j} C_{b i}\left(\left\langle\chi_{a}^{(1), R_{\lambda \alpha}} \mid \chi_{b}\right\rangle+\left\langle\chi_{a} \mid \chi_{b}^{(1), R_{\lambda \alpha}}\right\rangle\right) \\
& -S_{i j}^{(1), H_{\beta}} \sum_{a, b=1}^{N_{\text {bas }}} C_{a j} C_{b i}\left\langle\chi_{a}^{(1), R_{\lambda \alpha}} \mid \chi_{b}\right\rangle \\
& -U_{j i}^{(1), R_{\lambda \alpha}} * \sum_{a, b=1}^{N_{\text {bas }}} C_{a i} C_{b j}\left(\left\langle\chi_{a}^{(1), H_{\beta}} \mid \chi_{b}\right\rangle+\left\langle\chi_{a} \mid \chi_{b}^{(1), H_{\beta}}\right\rangle\right) \\
& -S_{i j}^{(1), R_{\lambda \alpha}} \sum_{a, b=1}^{N_{\text {bas }}} C_{a i} C_{b j}\left\langle\chi_{a} \mid \chi_{b}^{(1), H_{\beta}}\right\rangle
\end{aligned}
$$

The summations in the first and third terms of Eq. (54) are the $S_{j i}^{(1), R_{\lambda \alpha}}$ and $S_{i j}^{(1), H_{\beta}}$ matrix elements, respectively. Thus, Eq. (54) becomes:

$$
\begin{aligned}
& -U_{j i}^{(1), H_{\beta}{ }^{*}} S_{j i}^{(1), R_{\lambda \alpha}}-U_{j i}^{(1), R_{\lambda \alpha}{ }^{*}} S_{i j}^{(1), H_{\beta}} \\
& -S_{i j}^{(1), H_{\beta}} \sum_{a, b=1}^{N_{\mathrm{bas}}} C_{a j} C_{b i}\left\langle\chi_{a}^{(1), R_{\lambda \alpha}} \mid \chi_{b}\right\rangle
\end{aligned}
$$


$-S_{i j}^{(1), R_{\lambda \alpha}} \sum_{a, b=1}^{N_{\text {bas }}} C_{a i} C_{b j}\left\langle\chi_{a} \mid \chi_{b}^{(1), H_{\beta}}\right\rangle$

Using Eq. (22) the components of the fourth term in Eq. (25) due to a pair of occ-occ MOs $i$ and $j$ can be written:

$$
\begin{aligned}
& U_{i j}^{(1), R_{\lambda \alpha}} U_{i j}^{(1), H_{\beta}}+U_{j i}^{(1), R_{\lambda \alpha}} U_{j i}^{(1), H_{\beta}} \\
& =U_{i j}^{(1), R_{\lambda \alpha}} U_{i j}^{(1), H_{\beta}}+\left(-U_{i j}^{(1), R_{\lambda \alpha} *}-S_{j i}^{(1), R_{\lambda \alpha}}\right) \\
& \quad \times\left(-U_{i j}^{(1), H_{\beta}{ }^{*}}-S_{j i}^{(1), H_{\beta}}\right) \\
& =U_{i j}^{(1), R_{\lambda \alpha} *} S_{j i}^{(1), H_{\beta}}+S_{j i}^{(1), R_{\lambda \alpha}} U_{i j}^{(1), H_{\beta}{ }^{*}}+S_{j i}^{(1), R_{\lambda \alpha}} S_{j i}^{(1), H_{\beta}}
\end{aligned}
$$

By adding the results of Eqs. (55) and (56) we obtain the final expression for the contributions to $I_{\alpha \beta}^{\lambda}$ from of the last three terms of Eq. (25) due to a pair of occ-occ MOs $i$ and $j$ :

$$
\begin{aligned}
& -S_{j i}^{(1), R_{\lambda \alpha}} S_{j i}^{(1), H_{\beta}}-S_{i j}^{(1), H_{\beta}} \sum_{a, b=1}^{N_{\text {bas }}} C_{a j} C_{b i}\left\langle\chi_{a}^{(1), R_{\lambda \alpha}} \mid \chi_{b}\right\rangle \\
& -S_{i j}^{(1), R_{\lambda \alpha}} \sum_{a, b=1}^{N_{\text {bas }}} C_{a i} C_{b j}\left\langle\chi_{a} \mid \chi_{b}^{(1), H_{\beta}}\right\rangle
\end{aligned}
$$

As can be seen from Eq. (57) the off-diagonal matrix elements of the occ-occ blocks of both $U^{(1)}$ matrices are not needed for the calculation of the electronic contribution of the atomic axial tensor $\left(I_{\alpha \beta}^{\lambda}\right)$. Equation (22) is not equivalent with $U_{i j}^{(1)}=-\frac{1}{2} S_{i j}^{(1)}$ and $U_{j i}^{(1)^{*}}=-\frac{1}{2} S_{i j}^{(1)}$. Nevertheless, the same result may be obtained by substituting these expression into Eq. (48) and the first two terms of Eq. (56).

\section{References}

1. Stephens PJ (1987) Gauge dependence of magnetic dipole transition moments and rotational strength. J Phys Chem 91:1712-1715

2. Stephens PJ (1985) Theory of vibrational circular dichroism. J Phys Chem 89:748-752

3. Amsterdam Density Functional program. Theoretical Chemistry, Vrije Universiteit, Amsterdam. URL: http://www.scm. com

4. te Velde G, Bickelhaupt FM, Baerends EJ, Guerra CF, van Gisbergen SJA, Snijders JG, Ziegler T (2001) Chemistry with ADF. J Comput Chem 22:931-967

5. Boerrigter PM, te Velde G, Baerends EJ (1988) Threedimensional numerical integration for electronic structure calculations. Int J Quantum Chem 33:87-113

6. te Velde G, Baerends EJ (1992) Numerical integration for polyatomic systems. J Comp Phys 99:84-98

7. Kool ET (1998) Recognition of DNA, RNA, and proteins by circular oligonucleotides. Acc Chem Res 31:502-510
8. Kelly JW (1997) Amyloid fibril formation and protein misassembly: a structural quest for insights into amyloid and prion diseases. Structure 5:595-600

9. Furche F, Ahlrichs R, Wachsmann C, Weber E, Sobanski A, Vögtle F, Grimme S (2000) Circular dichroism of helicenes investigated by time-dependent density functional theory. J Am Chem Soc 122:1717-1724

10. Bürgi T, Urakawa A, Behzadi B, Ernst KH, Baiker A (2004) The absolute configuration of heptahelicene: a VCD spectroscopy study. New J Chem 28:332-334

11. Xu Y, Zhang YX, Sugiyama H, Umano T, Osuga H, Tanaka $\mathrm{K}(2004)(P)$-Helicene displays chiral selection in binding to Z-DNA. J Am Chem Soc 126:6566-6567

12. Herrmann C, Ruud K, Reiher M (2006) Can Raman optical activity separate axial from local chirality? A theoretical study of helical deca-alanine. ChemPhysChem 7:2189-2196

13. Buckingham AD, Fowler PW, Galwas PA (1987) Velocitydependent property surfaces and the theory of vibrational circular dichroism. Chem Phys 112:1-14

14. Amos RD, Handy NC, Jalkanen KJ, Stephens PJ (1987) Efficient calculation of vibrational magnetic dipole transition moments and rotational strength. Chem Phys Lett 133:21-26

15. Gerratt J, Mills I (1968) Force constants and dipole moment derivatives of molecules from perturbed Hartree-Fock calculations. I. J Chem Phys 49:1719-1729

16. Pople JA, Krishnan R, Schlegel HB, Binkley JS (1979) Derivative Studies in Hartree-Fock and Møller-Plesset theories. Int J Quant Chem Quant Chem Symp 13:225-241

17. McWeeny RJ (1964) Methods of molecular quantum mechanics, 2nd edn. Academic, New York

18. Ditchfield R (1974) Self-consistent perturbation theory of diamagnetism. Gauge-invariant LCAO method for NMR chemical shifts. Mol Phys 27:789-807

19. Schreckenbach G, Ziegler T (1995) Calculation of NMR shilding tensors using gauge-including atomic orbitals and modern density functional theory. J Phys Chem 99:606-611

20. Bak KL, Jorgensen $P$, Helgaker $T$, Ruud $K$, Jorgen $H$, Jensen Aa (1993) Gauge-origin independent multiconfigurational self-consistent theory for vibrational circular dichroism. J Chem Phys 98:8873-8887

21. Wolff SK (2005) Analytical second derivatives in the Amsterdam density functional package. Int $\mathrm{J}$ Quantum Chem 104:645-659

22. Becke AD (1988) Density-functional exchange-energy approximation with correct asymptotic behavior. Phys Rev A 38:3098-3100

23. Perdew JP (1986) Density-functional approximation for the correlation energy of the inhomogeneous electron gas. Phys Rev B 33:8822-8824

24. Handy NC, Cohen AJ (2001) Left-right correlation energy. Mol Phys 99:403-412

25. Hamprecht FA, Cohen AJ, Handy NC (1988) Development and assessment of new exchange-correlation functionals. J Chem Phys 109:6264-6271

26. Lee C, Yang W, Parr RG (1988) Development of the Colle-Salvetti correlation-energy formula into a functional of the electron density. Phys Rev B 37:785-789

27. van Lenthe E, Baerends EJ (2003) Optimized Slater-Type basis sets for elements 1-118. J Comput Chem 24:1142-1156

28. Jalkanen KJ, Kawiecki RW, Stephens PJ, Amos RD (1990) Basis set and gauge dependence of ab initio calculations of vibrational circular strengths. J Phys Chem 94:7040-7055

29. Jalkanen KJ, Stephens PJ, Amos RD, Handy NC (1988) Gauge dependence of vibrationl rotational strength: NHDT. J Phys Chem 92:1781-1785 
30. Stephens PJ, Jalkanen KJ, Amos RD, Lazzeretti P, Zanasi R (1990) Ab initio calculations of atomic polar and axial tensors for $\mathrm{HF}, \mathrm{H}_{2} \mathrm{O}, \mathrm{NH}_{3}$ and $\mathrm{CH}_{4}$. J Phys Chem 94:1811-1830

31. Yang D, Rauk A (1992) Vibrational circular dichroism intensities: AB inito vibronic coupling theory unsing the distributed origin gauge. J Chem Phys 97:6517-6534

32. Stephens PJ, Ashvar CS, Devlin FJ, Cheesman JR, Frisch MJ (1996) Ab initio calculation of atomic axial tensors and vibrational rotational strengths using density functional theory. Mol Phys 89:579-594

33. Devlin FJ, Stephens PJ, Cheeseman JR, Frisch MJ (1997) Ab initio prediction of vibrational absorbtion and circular dichroism spectra of chiral natural products using density functional theory: $\alpha$-Pinene. J Phys Chem A 101:9912-9924

34. Neugebauer J, Hess BA (2003) Fundamental vibrational frequencies of small polyatomic molecules from density functional calculations and vibrational perturbation theory. J Chem Phys 118:7215-7225

35. Reiher M, Neugebauer J, Hess BA (2003) Quantum chemical calculation of Raman intensities for large molecules: the photoisomerization of $\left[\left\{\mathrm{Fe}^{\prime} \mathrm{S}_{4} \text { ' }\left(\mathrm{PR}_{3}\right)\right\}_{2}\left(\mathrm{~N}_{2} \mathrm{H}_{2}\right)\right]\left(\right.$ ' $\mathrm{S}_{4}{ }^{, 2-}=$ 1,2-bis(2-mercaptophenylthio)ethane(2-)). Z Physik Chem 217:91-103

36. Neugebauer J, Baerends EJ, Efremov EV, Ariese F, Gooijer C (2005) Combined theoretical and experimental deep-UV resonance Raman studies of substituted pyrenes. J Phys Chem A 109:2100-2106

37. Aamouche A, Devlin FJ, Stephens PJ (2000) Structure, vibrational absorbtion and circular dichroism spectra, and absolute configuration of Troger's base. J Am Chem Soc 122:2346-2354

38. Kokalj A (1999) XCRYSDEN-a new program for displaying crystalline structures and electron densities. J Mol Graph Modeling 17:176-179
39. Becke AD (1983) Density-functional thermochemistry. III. The role of exact exchange. J Chem Phys 98:5648-5652

40. Stephens PJ, Devlin FJ, Chabalowski CF, Frisch MJ (1994) $\mathrm{Ab}$ initio calculation of vibrational absorption and circular dichroism spectra using density functional force fields. J Phys Chem 98:11623-11627

41. Freedman TD, Cao X, Young DA, Nafie LA (2002) Densiy functional theory calculations of vibrational circular dichroism in transition metal complexes: identification of solution conformations and mode of chloride ion association for (+)-Tris(ethylenediaminato)cobalt(III)+. J Phys Chem A 106:3560-3565

42. Kuppens T, Tollenaere JP, Langenaeker W, Bultinck P (2003) Determination of the stereochemistry of 3-Hydroxymethyl2,3-dihydro-[1.4]dioxino[2,3-b-]-pyridine by vibrational circular dichroism and the effect of DFT integration grid. J Phys Chem A 107:542-553

43. StephensPJ, Devlin FJ (2000) Determination of the structure of chiral molecules using Ab initio vibrational circular dichroism spectroscopy. Chirality 12:172-179

44. Devlin FJ, Stephens PJ, Cheeseman JR, Frisch MJ (1997) $\mathrm{Ab}$ initio prediction of vibrational absorption and circular dichroism spectra of chiral natural products using density functional theory: camphor and fenochone. J Phys Chem A 101:6311-6333

45. Wang F, Polavarapu PL, Schurug V, Schmidt R (2002) Absolute configuration and conformational analysis of a degradation product of inhalation anesthetic sevoflurane: a vibrational circular dichroism study. Chirality 14:618-624 